Interfaces and Free Boundaries 14 (2012), 65-103

DOI $10.4171 / \mathrm{IFB} / 274$

\title{
Phase transitions in presence of surfactants: From discrete to continuum
}

\author{
ROBERTO ALICANDRO \\ DAEIMI, Università di Cassino, via Di Biasio, 03043 Cassino (FR), Italy \\ E-mail: alicandr@unicas.it \\ Marco CicAlese \\ Dipartimento di Matematica e Applicazioni “R. Caccioppoli”, Università di Napoli “Federico II”, \\ via Cintia, 80126 Napoli, Italy \\ E-mail: cicalese@unina.it \\ LAURA SigALOTTI \\ Dipartimento di Matematica “G. Castelnuovo”, Università di Roma “La Sapienza”, Piazzale A. \\ Moro 2, 00185 Roma, Italy \\ E-mail:laura.sigalotti@gmail.com
}

[Received 29 June 2010 and in revised form 30 November 2011]

\begin{abstract}
We study by $\Gamma$-convergence the discrete-to-continuum limit of the Blume-Emery-Griffiths model describing the phase transition of a binary mixture in presence of a third surfactant phase. In the case of low surfactant concentration we study the dependence of the surface tension on the density of the surfactant and we describe the microstructure of the ground states. We then consider more general ( $n$-dimensional) energies modeling phase transitions in presence of different species of surfactants and, in the spirit of homogenization theory, we provide an integral representation result for their $\Gamma$ limit. As an application we study the ground states of these systems for prescribed volume fractions of the phases.
\end{abstract}

2010 Mathematics Subject Classification: Primary 49S05; Secondary 49J45, 49Q20, 82B24.

Keywords: Atomistic-to-continuum limit; spin systems; surface tension models; surfactants; $\Gamma$ convergence; calculus of variations.

\section{Introduction}

In recent years a great effort has been made in order to study the atomistic-to-continuum limit of several discrete systems and in particular of Ising-type spin systems (see, for example, [2, 3, 4, 6, 7, 8, 11, 12, 18, 19, 20, 30, 32] and [15, Chapter 11] for a review on this subject). Despite a simple structure, the energy functionals of such systems (depending on the choice of the order parameter, the range of interactions and the scaling properties) may model a great variety of physical phenomena. An instructive example in this respect is provided by the functional

$$
E_{\varepsilon}(u)=-\sum_{n . n .} \varepsilon^{2} u(a) \cdot u(b)
$$

which is the energy of a two-dimensional system of interacting spins through their nearest-neighbor (n.n.) bonds. Here the function $u$ is defined on the points of a $\varepsilon$-lattice and takes values either in $\{ \pm 1\}$ or in $S^{1}$. The energy accounts for the interactions among nearest neighbors (n.n.); i.e., 
$a, b \in \varepsilon \mathbf{Z}^{2} \cap \Omega$ such that $|a-b|=\varepsilon\left(\Omega \subset \mathbf{R}^{2}\right.$ being a bounded open set). The analysis of such a system can be performed both when $u$ takes values either in $\{ \pm 1\}$ and in $S^{1}$ (in the latter case $u(a) \cdot u(b)$ denotes the scalar product in $\mathbf{R}^{2}$ ). On one hand, in the case $u \in\{ \pm 1\}$ it has been proved in [2] that the discrete-to-continuum limit, as $\varepsilon \rightarrow 0$, of suitable power scaling of (1.1) resembles that of a Cahn-Hillard type functional. Namely, it leads to a class of anisotropic surface tension energies of the form $\int_{\partial E} \varphi\left(v_{E}\right) d H^{1}$, where $v_{E}$ stands for the inner normal to $\partial E$ which, in turns, represents the interface between the $\{u=+1\}$ phase and the $\{u=-1\}$ phase. On the other hand, the vectorial case $u \in S^{1}$ leads to a completely different class of continuum limits. In [4] it has been proved that the asymptotic analysis, as $\varepsilon \rightarrow 0$, of a suitable logarithmic scaling of (1.1) resembles that of a complex Ginzburg-Landau functional. In particular, it leads to a limit energy model for the formation of vortex-type singularities described through the measure $\mu=\sum_{i=1}^{N} d_{i} \delta_{x_{i}}\left(x_{i} \in \Omega\right.$ standing for the location of the $i$-th singularity and $d_{i} \in \mathbf{Z}$ for its topological degree) whose energy is of the type $|\mu|(\Omega)$.

In this paper we will prove that a variant of the energies in (1.1) leads to a class of continuum functionals different from the two mentioned above. The model we consider can be described as follows. Given $\Omega \subset \mathbf{R}^{2}$ a bounded open set, we fix the two dimensional square lattice $\varepsilon \mathbf{Z}^{2} \cap \Omega$ and we consider a ternary system driven by an energy defined on functions parameterized on the points of the lattice and taking only three different values (for simplicity, $-1,0,1$ ). In this framework, the values of $u$ are usually thought to describe three admissible phases of the system. For a given configuration of particles, the free energy $E_{\varepsilon}$ of the system is given by

$$
E_{\varepsilon}(u)=\sum_{n . n .} \varepsilon^{2}\left(-u(a) u(b)+k(u(a) u(b))^{2}\right) .
$$

Here $k>0$ is the quotient between the so called bi-quadratic and quadratic exchange interaction strengths. The range of $k$, jointly with the scaling of the energy, will be chosen later as a result of a heuristic argument. This heuristic argument will justify the choice of the functional in (1.2) as the free energy of a system where the two phases $\{ \pm 1\}$ coexist in presence of the phase $\{0\}$ representing a substance called surfactant (a contraction for surface-active-agent) which, by being absorbed onto the interfaces, may significantly reduce the surface tension of the system.

A variational description of the effects caused by the presence of surfactants in phase separation phenomena has been developed by modeling the physical system either as a continuum or as a discrete. Among the continuum theories, a first attempt to model phase transitions in presence of surfactants has been made by Laradji-Guo-Grant-Zuckermann in [27] and [28] (several generalizations have been further considered by Gompper and Schick in [26]) who proposed a variational model involving a two order parameters Ginzburg-Landau functional. Here one order parameter represents the local difference of density of the two phases (as in the standard Cahn-Hillard model in the gradient theory of phase transitions) while the other one represents the local surfactant density. The two order parameters are energetically coupled to favor the segregation of the surfactant at the phase interface. The coarse-graining analysis of this model has been performed through $\Gamma$ convergence methods by Fonseca, Morini and Slastikov in [25] (the mathematical analysis of more general continuum models is the object of [1]). Concerning the discrete models, many of them use some variation of that in (1.2) which has been introduced by Blume, Emery and Griffiths (BEG) in [13] (see also [26] and the references therein). This model has been also object of interesting studies in the context of equilibrium statistical mechanics (see [24] for an exhaustive mean-field approach) 
and has been used to describe several ternary systems (e.g., solid-liquid-gas systems, semiconductor alloys, electronic conduction models).

In this paper we will perform a $\Gamma$-limit analysis of these energies and, as a result, we will be able to describe the behavior of the ground states of the BEG system as $\varepsilon$ tends to 0 . More in details, upon identifying arrays $\{u(a)\}, a \in \varepsilon \mathbf{Z}^{2} \cap \Omega$ with their piecewise-constant interpolations, the energies $E_{\varepsilon}$ can be interpreted as defined on (a subset of) $L^{1}(\Omega)$, and can therefore undergo a process of $\Gamma$-limit in that framework. As $\varepsilon$ tends to 0 , the $\Gamma$-limit $E$ of $E_{\varepsilon}$ is particularly simple, only giving the trivial constraint $|u| \leqslant 1$, and the constant (minimum) value $2|\Omega|(-1+k) \wedge 0$, achieved by one of the three uniform states $u=-1, u=+1$ and $u=0$. By choosing $k<1$ we set the uniform states $u= \pm 1$ to be the ground states. Note that, with this choice, the asymptotic analysis of the energy $E_{\varepsilon}$ summarizes the fact that a sequence $\left(u_{\varepsilon}\right)_{\varepsilon}$ can arbitrarily mix the uniform states -1 and 1 at a mesoscopic scale with variation in energy from the value of the uniform states which is negligible as $\varepsilon \rightarrow 0$ (the asymptotic analysis of the bulk scaling of more general spin-type models has been performed in [6]). Thus, in order to have a better description of the ground states, in the spirit of development by $\Gamma$-convergence (see [21] for a general treatment of this topic and also [2, $5,16,17])$, we select sequences that realize the minimum value with a sharper precision; i.e., such that

$$
E_{\varepsilon}\left(u_{\varepsilon}\right)=c_{\varepsilon}+O(\varepsilon)
$$

where we have denoted by $c_{\varepsilon}$ the absolute minimum of $E_{\varepsilon}$, that is $c_{\varepsilon}=\sum_{\text {n.n. }} \varepsilon^{2}(k-1)$. For such configurations the limit states $u$ will take the values \pm 1 only and the limit energy will be an interfacial type energy which is interpreted as the surface tension of the system which undergoes a phase separation phenomenon between the phases $\{u=-1\}$ and $\{u=+1\}$. Thus, at this scaling, it is necessary to further specify the values of the parameter $k$ in such a way that the phase 0 can be actually considered as a surfactant phase (meaning that it contributes to lower the surface tension). In particular it can be easily showed (see Section 3 ) that, for $\frac{1}{3}<k<1$, the energy for a transition from $a-1$ phase to $a+1$ phase is lowered if the surfactant particles are at the interface. With such a choice, the 0 phase can be rightly considered a surfactant phase and, for this reason, the previous scaling, in which in addition the measure of the phase 0 vanishes, is usually called the low surfactant concentration regime. We are interested in this scaling regime. Thus the scaled family of functionals we have to study is

$$
E_{\varepsilon}^{(1)}(u):=\frac{E_{\varepsilon}(u)-c_{\varepsilon}}{\varepsilon}=\sum_{n . n .} \varepsilon\left(1-u(a) u(b)-k\left(1-(u(a) u(b))^{2}\right) .\right.
$$

Observe that, within this scaling, the interaction energy for two particles of the same type -1 or +1 is zero, while the interaction of a surfactant particle 0 with respect to all the other particles is repulsive, its cost being the positive value $1-k$. For this reason it is also said that the BEG functional describes a repulsive surfactant model. In Theorem 3.2 we show that $E_{\varepsilon}^{(1)} \Gamma$-converges (in the $L^{1}(\Omega)$-topology) to the interfacial-type energy functional

$$
E^{(1)}(u)=\int_{S(u)} \psi\left(v_{u}\right) d H^{1}
$$

where $u \in B V(\Omega ;\{ \pm 1\}), \psi(v)=(1-k)\left(3\left|v_{1}\right| \vee\left|v_{2}\right|+\left|v_{1}\right| \wedge\left|v_{2}\right|\right)$ denotes the anisotropic surface tension of the model, $S(u)$ is the (essential) interface between the sets $\{u=1\}$ and $\{u=-1\}$ and $v_{u}$ is the measure theoretic inner normal to $S(u)$. 
As we have already observed, in the topology we have chosen, the limit order parameter $u$ does not carry any information about the surfactant phase. The role of the surfactant becomes clear when one looks at the minimizing microstructure leading to the computation of the limiting surface density $\psi$. In this direction, a natural further step in the analysis of the BEG model is the dependence of the surface tension of the continuum limit on the concentration of the surfactant. There is a large literature on this subject, both from the physical and the chemical point of view (see for example [26] and [29]). However no rigorous description of the microscopic geometry of the surfactant at the interface is present in literature and, as far as we know, all the attempts done to study this problem are based on numerical computations or on heuristic arguments. In order to rigorously address this problem we need to go beyond the standard formulation of the BEG model and let the energy functional of the system depend explicitly on the distribution of the surfactant particles. To this end we set

$$
I_{0}(u)=\left\{a \in \Omega_{\varepsilon}: u(a)=0\right\},
$$

and we introduce the following surfactant measure

$$
\mu(u)=\sum_{a \in I_{0}(u)} \varepsilon \delta_{a} .
$$

Then, with a slight abuse of notation, we can extend $E_{\varepsilon}^{(1)}$ to $L^{1}(\Omega) \times m_{+}(\Omega) \rightarrow[0,+\infty]$ as

$$
E_{\varepsilon}^{(1)}(u, \mu)= \begin{cases}E_{\varepsilon}^{(1)}(u) & \text { if } \mu=\mu(u) \\ +\infty & \text { otherwise. }\end{cases}
$$

We observe that, in this discrete setting, the way we extend the functionals in order to track the energy of the surfactants is by decoupling the order parameter of the model instead of adding another variable as it has been done when dealing with continuum models (see [25] and [1]). We then endow the space $L^{1}(\Omega) \times m_{+}(\Omega)$ with the topology $\tau_{1} \times \tau_{2}$ where $\tau_{1}$ denotes the strong topology in $L^{1}(\Omega)$ and $\tau_{2}$ denotes the weak*-topology in the space of non-negative bounded Radon measures $\eta_{+}(\Omega)$ and, by performing the $\Gamma$-limit with respect to this topology, in Theorem 3.3 we prove that $E_{\varepsilon}^{(1)}$ $\Gamma$-converges to the functional $E^{(1)}: L^{1}(\Omega) \times m_{+}(\Omega) \rightarrow[0,+\infty]$ defined as

$$
E^{(1)}(u, \mu)= \begin{cases}\int_{S(u)} \varphi\left(\frac{d \mu}{d \mathcal{H}^{1}\lfloor S(u)}, v_{u}\right) d \mathcal{H}^{1}+(2 k-2)\left|\mu^{s}\right|(\Omega) & \text { if } u \in B V(\Omega ;\{ \pm 1\}), \\ +\infty & \text { otherwise, }\end{cases}
$$

where we have set $\mu^{s}:=\mu-\frac{d \mu}{d \mathcal{H}^{1}\lfloor S(u)} \mathcal{H}^{1}\left\lfloor S(u)\right.$ and where the function $\varphi: \mathbf{R} \times S^{1} \rightarrow[0,+\infty)$ is computed explicitly and its graph is reported in Figure 2. As it is clear from the graph of $\varphi$, a threshold phenomenon depending on $v$ occurs at the phase interface. Indeed, for a given $v \in S^{1}$ the surface tension $\varphi(z, v)$ decreases up to a certain value of the density $z$ of the surfactant, namely $z=\left|v_{1}\right| \vee\left|v_{2}\right|$. Increasing further the density of the surfactant the surface tension is constant, if the surfactants are not absorbed onto the interface (in this case the singular part of the surfactant measure is increased), or otherwise it increases. As an application of the previous $\Gamma$-convergence result, at the end of section 3 we address an optimization problem having as constraint the prescribed volume fractions of the different phases.

Inspired by the many different models of phase transitions in presence of surfactants studied in the physical/chemical literature, in Section 4 we push forward the previous analysis to the case 

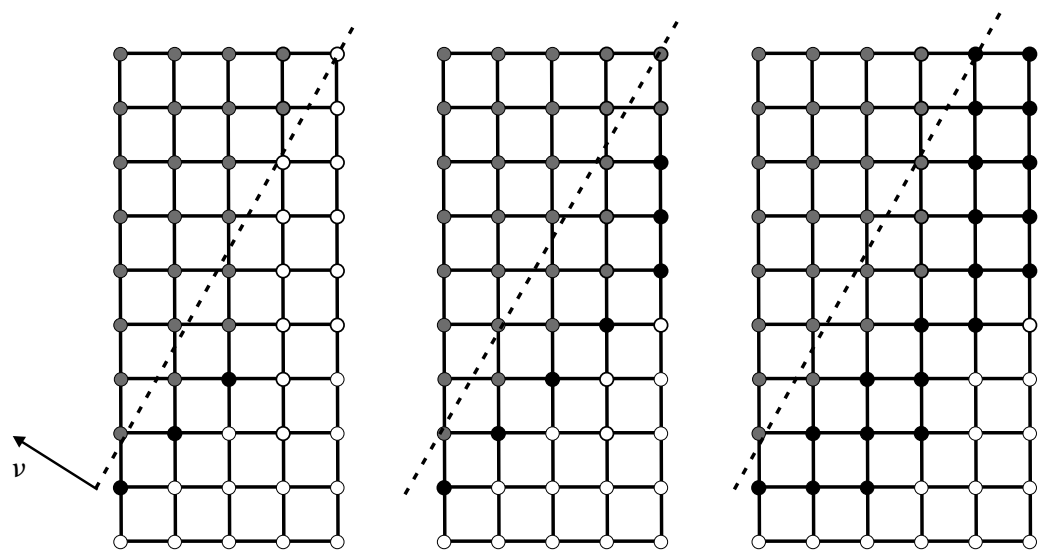

FIG. 1. The local microstructure of a ground state of the BEG model at a fixed straight interface (the dashed line normal to $v$ ) for three different values of the density of surfactants at the interface. Black, white and grey dots stand for the $0,+1$ and -1 values of the spin field $u$, respectively.

of a $n$-dimensional discrete systems driven by an energy accounting for quite general finite range pairwise interactions when different species of repulsive surfactant particles are present. For such a general system, in the spirit of homogenization theory, we obtain an integral representation result for the $\Gamma$-limit and we study some properties of its densities. More precisely, given $\Omega \subset \mathbf{R}^{n}$ and $u: \varepsilon \mathbf{Z}^{n} \cap \Omega \rightarrow K$ we define the functional $F_{\varepsilon}$ as

$$
F_{\varepsilon}(u)=\sum_{a, b \in \Omega_{\varepsilon},|a-b| \leqslant R \varepsilon} \varepsilon^{n-1} f\left(\frac{b-a}{\varepsilon}, u(a), u(b)\right),
$$

where $R>0$ is an interaction threshold, $K=\left\{m_{1}, m_{2}, s_{1}, s_{2}, \ldots, s_{M}\right\} \subset \mathbf{R}$ describes the finite number of phases in the system and $f: \mathbf{Z}^{n} \times K^{2} \rightarrow[0,+\infty)$ satisfies a sort of discrete isotropy condition (see Remark 4.1 and 4.5) and is such that $f(z, \cdot, \cdot)$ has $\left\{\left(m_{1}, m_{1}\right),\left(m_{2}, m_{2}\right)\right\}$ as absolute minimizers. As we have done in the case of the BEG model, to study the discrete-to-continuum limit of this discrete system we introduce the sets of points in $\varepsilon \mathbf{Z}^{n} \cap \Omega$ occupied by the different types of surfactant and suitable surfactant measures associated to them. For $l \in\{1,2, \ldots, M\}$ we set

$$
I_{l}(u):=\left\{a \in \Omega_{\varepsilon}: u(a)=s_{l}\right\}, \quad I(u):=\bigcup_{l=1}^{M} I_{l}(u)
$$

and we define

$$
\mu_{l}(u):=\sum_{a \in I_{l}(u)} \varepsilon^{n-1} \delta_{a}, \quad \mu(u)=\left\{\mu_{1}(u), \mu_{2}(u), \ldots, \mu_{M}(u)\right\} .
$$

After extending $F_{\varepsilon}$ to $L^{1}(\Omega) \times\left(m_{+}(\Omega)\right)^{M} \rightarrow[0,+\infty]$ as

$$
F_{\varepsilon}(u, \mu):= \begin{cases}F_{\varepsilon}(u) & \text { if } \mu=\mu(u) \\ +\infty & \text { otherwise }\end{cases}
$$


in Theorem 4.4 we prove that $F_{\varepsilon} \Gamma$-converge with respect to the $\tau_{1} \times \tau_{2}$ convergence (here $\tau_{1}$ denotes the strong convergence in $L^{1}(\Omega)$ and $\tau_{2}$ denotes the weak ${ }^{*}$-convergence in $\left.\left(m_{+}(\Omega)\right)^{M}\right)$ to the functional

$$
F(u, \mu)=\int_{S(u)} f_{\text {hom }}\left(\frac{d \mu}{d \mathcal{H}^{n-1}\lfloor S(u)}, v(u)\right) d \mathcal{H}^{n-1}+\int_{\Omega} g_{\text {hom }}\left(\mu^{s}\right)
$$

if $u \in B V\left(\Omega ;\left\{m_{1}, m_{2}\right\}\right), \mu=\frac{d \mu}{d \mathcal{H}^{n-1}\lfloor S(u)} \mathcal{H}^{n-1}\left\lfloor S(u)+\mu^{s}\right.$, and $+\infty$ otherwise. The limit densities $f_{\text {hom }}$ and $g_{\text {hom }}$ are given by two asymptotic homogenization formulas in (4.9) and (4.10) respectively. In order to derive the formula defining $g_{\text {hom }}$ we need to devise new homogenization arguments, by combining some abstract arguments of measure theory with a reflection construction which exploits the discrete isotropy assumption on the interaction densities (see Remark 4.5).

As a final remark, we want to underline that in the models we consider here the surfactants are described as scalar particles without internal structure. More general models are known (see [22, 31] and [26]) where the surfactants are described as polar molecules whose heads and tails interact differently with the same phase. In this case it is also known that the presence of surfactants in a mixture may lead to self-assembling and that a number of different, and even topologically non trivial, microstructures may appear. We hope that the analysis performed in this paper may provide the basis to address the discrete-to-continuum limit for these systems.

Acknowledgements. The work by Marco Cicalese was partially supported by the European Research Council under FP7, Advanced Grant n. 226234 "Analytic Techniques for Geometric and Functional Inequalities". This work is part of the third author's Ph.D. thesis at the Department of Mathematics of Sapienza, Università di Roma.

\section{Notation and preliminaries}

In what follows, given $x, y \in \mathbf{R}^{n}$ we denote by $(x, y)$ the usual scalar product in $\mathbf{R}^{n}$ and we set $|x|=\sqrt{(x, x)}$. Moreover we denote by $\|\cdot\|_{1}$ the $l_{1}$-norm in $\mathbf{R}^{n}$ defined as $\|x\|_{1}=\left|x_{1}\right|+\cdots+\left|x_{n}\right|$. Given $t>0$, we will denote by $[t]$ the integer part of $t$. For any measurable $A \subset \mathbf{R}^{n}$ we denote by $|A|$ the $n$-dimensional Lebesgue measure of $A$. Let $\Omega$ be a bounded open subset of $\mathbf{R}^{n}$ with Lipschitz boundary. For fixed $\varepsilon>0$ we consider the lattice $\varepsilon \mathbf{Z}^{n} \cap \Omega=: \Omega_{\varepsilon}$. Given $K \subset \mathbf{R}$ we denote by $Q_{\varepsilon}(\Omega ; K)$ the set of functions

$$
Q_{\varepsilon}(\Omega ; K):=\left\{u: \Omega_{\varepsilon} \rightarrow K\right\} .
$$

REMARK 2.1 A function $u \in Q_{\varepsilon}(\Omega ; K)$ will be identified with its piecewise-constant interpolation still denoted by $u$ and given by $u(x)=u\left(z_{x}^{\varepsilon}\right)$, where $z_{x}^{\varepsilon} \in \mathbf{Z}^{n}$ is the closest point to $x$ (which is uniquely defined up to a set of zero measure). In this definition, we set $u(z)=0$ if $z \in \varepsilon \mathbf{Z}^{n} \backslash \Omega$. In such a way $Q_{\varepsilon}(\Omega ; K)$ will be regarded as a subset in $L^{1}(\Omega)$.

We denote by $H^{n-1}$ the $n-1$-dimensional Hausdorff measure. Given $v=\left(v_{1}, \ldots, v_{n}\right) \in S^{n-1}$ we set

$$
Q_{v}:=\left(-r_{v}, r_{v}\right)^{n},
$$

where $r_{v}>0$ is such that

$$
\mathcal{H}^{n-1}\left(Q_{v} \cap \Pi_{v}\right)=1,
$$




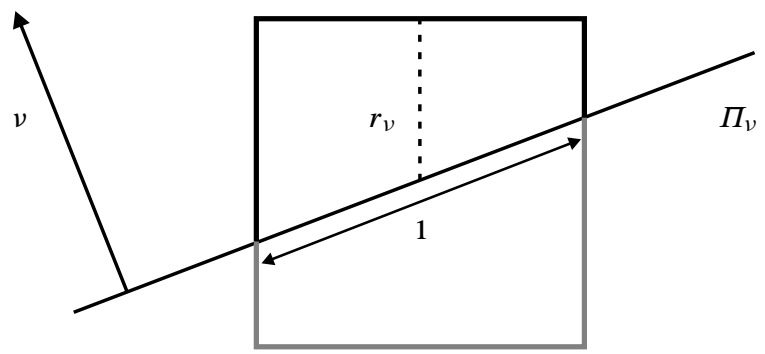

FIG. 2. The cube $Q_{\nu}$. In black $\partial^{+} Q_{\nu}$ and in grey $\partial^{-} Q_{\nu}$.

with $\Pi_{v}:=\left\{x \in \mathbf{R}^{n}:(x, v)=0\right\}$. We drop the dependence on $v$ whenever $v=e_{i}$ for $i \in$ $\{1,2, \ldots, n\}$ and we set $Q:=Q_{e_{i}}=\left(-\frac{1}{2}, \frac{1}{2}\right)^{n}$.

For any $T>0$ we set

$$
\partial^{ \pm}\left(T Q_{v}\right):=\left\{x \in \partial\left(T Q_{v}\right): \pm(x, v) \geqslant 0\right\}
$$

and then we introduce the discrete boundary of $T Q_{\nu}$, to be used in Section 4 as

$$
\partial_{\varepsilon}^{ \pm}\left(T Q_{v}\right):=\left\{a \in \varepsilon \mathbf{Z}^{n} \cap T Q_{v}:\left(a+[-R \varepsilon, R \varepsilon]^{n}\right) \cap \partial^{ \pm}\left(T Q_{v}\right) \neq \varnothing\right\},
$$

where $R>0$ is the parameter appearing in (4.1).

Next we recall some basic properties of $B V$ functions with values in a finite set (see [9] for a general exposition of the subject). Let $A$ be an open bounded subset of $\mathbf{R}^{n}$ and let $J$ be a finite subset of $\mathbf{R}$. We denote by $B V(A ; J)$ the set of measurable function $u: A \rightarrow J$ whose distributional derivative $D u$ is a measure with bounded total variation. We denote by $S(u)$ the jump set of $u$ and by $v_{u}(x)$ the measure theoretic inner normal to $S(u)$ at $x$, which is defined for $\mathcal{H}^{n-1}$ a.e. $x \in S(u)$.

For the reader's convenience we recall the following compactness result (see [9]).

THEOREM 2.2 Let $u_{k} \in B V(A ; J)$ such that

$$
\sup _{k} H^{n-1}\left(S\left(u_{k}\right)\right)<+\infty .
$$

Then there exists a subsequence (not relabelled) and $u \in B V(A ; J)$ such that $u_{k} \rightarrow u$ in the $L^{1}$ convergence.

If $Q$ is a cube we will denote by $B V^{\#}(Q ; J)$ the set of $Q$-periodic functions belonging to $B V_{\text {loc }}\left(\mathbf{R}^{n} ; J\right)$.

\section{The Blume-Emery-Griffiths model}

In this section we briefly introduce the Blume-Emery-Griffiths model which models phase transitions of water and oil in presence of surfactants.

\subsection{A brief description of the model: From bulk to surface scaling}

In its standard formulation the Blume-Emery-Griffiths model can be described as follows. Given a bounded open set $\Omega \subset \mathbf{R}^{2}$ with Lipschitz boundary, on the square lattice $\Omega_{\varepsilon}=\varepsilon \mathbf{Z}^{2} \cap \Omega$ we consider 
the set $Q_{\varepsilon}(\Omega ;\{ \pm 1,0\}):=\left\{u: \Omega_{\varepsilon} \rightarrow\{ \pm 1,0\}\right\}$ of those functions $u$ whose values on the sites of the lattice correspond to a particle of water (or oil) (in this framework the scale is not precisely specified and the term particle means more in general a molecule or an aggregate of molecules) for $u= \pm 1$ or of surfactant for $u=0$. We then introduce the family of energies $E_{\varepsilon}^{\text {latt }}(u): \mathbb{Q}_{\varepsilon}(\Omega ;\{ \pm 1,0\}) \mapsto \mathbf{R}$

$$
E_{\varepsilon}^{l a t t}(u)=\sum_{n . n .} \varepsilon^{2}\left(-u(a) u(b)+k\left((u(a) u(b))^{2}\right),\right.
$$

where $n . n$. means that the sum is performed over those $a, b \in \Omega_{\varepsilon}$ such that $|a-b|=\varepsilon$ and $k>0$ is a parameter which measures the strength of the quadratic with respect to the bi-quadratic interactions. The asymptotic analysis of such a family of energies, as $\varepsilon$ tends to 0 , is particularly simple and can be obtained through a dual lattice approach as in [2]. Indeed, by identifying the functions $u \in Q_{\varepsilon}(\Omega ;\{ \pm 1,0\})$ with their piecewise constant interpolations (see Remark 2.1), we first extend the energies $E_{\varepsilon}^{\text {latt }}$ in (3.1) to a functional $E_{\varepsilon}: L^{1}(\Omega) \rightarrow[0,+\infty]$ as

$$
E_{\varepsilon}(u)= \begin{cases}E_{\varepsilon}^{l a t t}(u) & \text { if } u \in Q_{\varepsilon}(\Omega ;\{ \pm 1,0\}), \\ +\infty & \text { otherwise }\end{cases}
$$

and then compute the $\Gamma$-limit of $\left(E_{\varepsilon}\right)$ with respect to the weak topology in $L^{1}(\Omega)$.

As a result one obtains that the following Theorem holds true:

THEOREM 3.1 The family $\left(E_{\varepsilon}\right) \Gamma$-converges with respect to the $L^{1}(\Omega)$-weak topology to the functional $E: L^{1}(\Omega) \rightarrow \mathbf{R} \cap\{+\infty\}$ defined as

$$
E(u)= \begin{cases}2|\Omega|(k-1) \wedge 0 & \text { if } u \in L^{1}(\Omega ;[-1,1]) \\ +\infty & \text { otherwise }\end{cases}
$$

Let us comment the previous result in the interesting case when $k<1$. In this regime the lattice energy has the two pure states $u= \pm 1$ as minimizers, and all the variations of the order parameter from these states are of order $\varepsilon^{2}$ in the discrete energy. This implies that, in the continuum limit, it is possible to obtain, with finite energy, any value of the order parameter $u$ in $[-1,+1]$ by arbitrarily mixing the two ground states on a mesoscopic scale $\varepsilon<<\delta<<1$. In particular this allows the energy of a phase separation to be negligible. More precisely the energy for a phase transition from a bulk -1 phase to a bulk +1 phase separated by an interface of finite length has an energy of order $\varepsilon$ and suggests the correct scaling to track the energetic behavior of a phase separation phenomenon. Observing that the absolute minimum value at scale $\varepsilon$ is precisely given by

$$
m_{\varepsilon}=\sum_{n . n .} \varepsilon^{2}(k-1),
$$

in order to have a more detailed description of the ground states we select those configurations described by functions $u_{\varepsilon}$ that realize the minimum value with a sharper precision; i.e., such that

$$
E_{\varepsilon}\left(u_{\varepsilon}\right)=m_{\varepsilon}+O(\varepsilon)
$$

In other words this amounts to study the family of discrete energies $E_{\varepsilon}^{(1)}: L^{1}(\Omega) \rightarrow[0,+\infty]$ defined as

$$
E_{\varepsilon}^{(1)}(u):=\frac{E_{\varepsilon}(u)-m_{\varepsilon}}{\varepsilon},
$$


that is

$$
E_{\varepsilon}^{(1)}(u)= \begin{cases}\sum_{n . n .} \varepsilon\left(1-u(a) u(b)-k\left(1-(u(a) u(b))^{2}\right)\right. & \text { if } u \in Q_{\varepsilon}(\Omega ;\{0, \pm 1\}), \\ +\infty & \text { otherwise. }\end{cases}
$$

Within this scaling, the measure of the surfactant phase has to be negligible in the continuum limit. More precisely, it is easily seen that, since each interaction with a surfactant particle pays a positive energy $\varepsilon(1-k)$, the following estimate

$$
E_{\varepsilon}^{(1)}(u) \geqslant \#\left\{a \in \Omega_{\varepsilon}: u(a)=0\right\} \varepsilon(1-k) \geqslant \frac{C}{\varepsilon}|\{x \in \Omega: u(x)=0\}|
$$

implies that the measure of the surfactant phase scales as $\varepsilon$. As a result, the finite energy states $u$ will only take the values \pm 1 . It is now possible to further specify the values of the parameter $k$ in such a way that the phase 0 can be actually considered a surfactant phase, meaning that it lowers the surface tension in the continuum limit. To obtain such values of $k$ we can proceed by computing the energy for a transition from -1 to +1 in the simplest case when the interface is a straight line in one of the directions of the lattice (say $e_{1}$ ). Suppose for simplicity that $\Omega=Q$ and that the interface is the set $\left\{x \in Q:\left(x, e_{2}\right)=0\right\}$. Our estimates are obtained by comparing the energy for such a macroscopic transition when the microscopic structure is either given by $u_{\varepsilon}$ or by $v_{\varepsilon}$, where

$$
u_{\varepsilon}(a)= \begin{cases}+1 & \text { if }\left(a, e_{2}\right) \geqslant 0 \\ -1 & \text { otherwise }\end{cases}
$$

and

$$
v_{\varepsilon}(a)= \begin{cases}+1 & \text { if }\left(a, e_{2}\right)>0 \\ 0 & \text { if }\left(a, e_{2}\right)=0 \\ -1 & \text { otherwise }\end{cases}
$$

It holds that

$$
\begin{aligned}
& E_{\varepsilon}^{(1)}\left(u_{\varepsilon}\right)=2+o(1), \\
& E_{\varepsilon}^{(1)}\left(v_{\varepsilon}\right)=3(1-k)+o(1) .
\end{aligned}
$$

Imposing now that the microstructure with the surfactant lowers the interface energy means to impose that $E_{\varepsilon}^{(1)}\left(v_{\varepsilon}\right)<E_{\varepsilon}^{(1)}\left(u_{\varepsilon}\right)$. This turns out to imply the condition $k>\frac{1}{3}$. Such an estimate is indeed proven to be sufficient to treat the case of a more general interface, as it is shown in Theorem 3.2 .

Finally we remark that the previous heuristic derivation of the range of the parameter $k$ leads us to call such a regime the low surfactant concentration regime. In this regime it can be also justified the negligibility, in the present model, of other terms originally present in the BEG energy, such as the chemical potential of the surfactant phase, which in a general situation cannot be ignored (see [29]).

THEOREM 3.2 Let $\frac{1}{3}<k<1$ and let $\left(E_{\varepsilon}^{(1)}\right)_{\varepsilon}$ be the family of functionals defined as in (3.2). Then we have 
(i) for any sequence $\left(u_{\varepsilon}\right) \subseteq L^{1}(\Omega)$ such that

$$
\sup _{\varepsilon} E_{\varepsilon}^{(1)}\left(u_{\varepsilon}\right) \leqslant C<+\infty
$$

there exist $\left(u_{\varepsilon_{k}}\right)_{k \in \mathbf{N}}$ and $u \in B V(\Omega ;\{ \pm 1\})$ such that

$$
u_{\varepsilon_{k}} \rightarrow u \text { for } k \rightarrow+\infty
$$

with respect to the $L^{1}(\Omega)$-topology;

(ii) the family of functionals $\left(E_{\varepsilon}^{(1)}\right) \Gamma$-converges with respect to the $L^{1}(\Omega)$-topology to the functional $E^{(1)}: L^{1}(\Omega) \rightarrow[0,+\infty]$ defined by

$$
E^{(1)}(u)= \begin{cases}\int_{S(u)} \psi\left(v_{u}\right) d \mathcal{H}^{1} & \text { if } u \in B V(\Omega ;\{ \pm 1\}) \\ +\infty & \text { otherwise, }\end{cases}
$$

where the function $\psi: S^{1} \rightarrow[0,+\infty)$ is given by

$$
\psi(v)=(1-k)\left(3\left|v_{1}\right| \vee\left|v_{2}\right|+\left|v_{1}\right| \wedge\left|v_{2}\right|\right) .
$$

Proof. We will derive the proof as a consequence of Theorem 3.3. The compactness result stated in (i) is a straightforward consequence of the analogous result stated in Theorem 3.3 (i). In order to prove the $\Gamma$-lim inf inequality, let us first note that the function $\varphi(\cdot, \cdot)$ defined in (3.6) satisfies

$$
\min \left\{\varphi(z, v): z \in \mathbf{R}_{+}\right\}=\varphi\left(\left|v_{1}\right| \vee\left|v_{2}\right|, \nu\right)=\psi(\nu) .
$$

Hence the functional $E^{(1)}(\cdot, \cdot)$ defined in (3.5) satisfies

$$
E^{(1)}(u, \mu) \geqslant E^{(1)}(u), \quad \forall(u, \mu) \in B V(\Omega ;\{ \pm 1\}) \times M^{+}(\Omega)
$$

Let $u_{\varepsilon} \rightarrow u$ in $L^{1}(\Omega)$. By Theorem 3.3 (i), we may assume that $\mu\left(u_{\varepsilon}\right) \rightarrow \mu$ weakly in the sense of measures. Then

$$
\liminf _{\varepsilon} E_{\varepsilon}^{(1)}\left(u_{\varepsilon}\right) \geqslant E^{(1)}(u, \mu) \geqslant E^{(1)}(u) .
$$

By a density argument it suffices to prove the $\Gamma$-lim sup inequality for a function $u$ with a polyhedral jump set. Since the construction is local it is enough to consider $u=u_{v}$, where $u_{v}$ is defined in (5.5). For such a function the optimizing sequence is given by $v_{z, v}(\dot{\bar{\varepsilon}})$, where $v_{z, v}$ is defined in (5.6), with $z=\left|v_{1}\right| \vee\left|v_{2}\right|$.

\subsection{Low concentration of surfactants: discrete-to-continuum limit}

As we have seen in the previous section, in the topology we have chosen, the limit order parameter $u$ does not carry any information about the surfactant phase. Actually the role of the surfactant becomes clear when one looks at the minimizing microstructure leading to the computation of the limiting surface density $\psi$. A natural further step in the analysis of the BEG model is the dependence of the surface tension of the continuum limit on the concentration of the surfactant. To make it explicit, we need to go beyond the standard formulation of the BEG model and we let the energy 
functional of the system depend explicitly on the distribution of the surfactant particles. To this end, for all $u \in Q_{\varepsilon}(\Omega ;\{0, \pm 1\})$ we set

$$
I_{0}(u)=\left\{a \in \Omega_{\varepsilon}: u(a)=0\right\},
$$

and we introduce the following surfactant measure

$$
\mu(u)=\sum_{a \in I_{0}(u)} \varepsilon \delta_{a} .
$$

Then, with a slight abuse of notation, we can extend $E_{\varepsilon}^{(1)}$ to a functional $E_{\varepsilon}^{(1)}: L^{1}(\Omega) \times m_{+}(\Omega) \rightarrow$ $[0,+\infty]$ as

$$
E_{\varepsilon}^{(1)}(u, \mu)= \begin{cases}E_{\varepsilon}^{(1)}(u) & \text { if } u \in Q_{\varepsilon}(\Omega ;\{0, \pm 1\}), \mu=\mu(u), \\ +\infty & \text { otherwise. }\end{cases}
$$

We endow the space $L^{1}(\Omega) \times m_{+}(\Omega)$ with the topology $\tau_{1} \times \tau_{2}$ where $\tau_{1}$ denotes the strong topology in $L^{1}(\Omega)$ and $\tau_{2}$ denotes the weak ${ }^{*}$-topology in the space of non-negative bounded Radon measures $m_{+}(\Omega)$.

The following Theorem holds true.

THEOREM 3.3 Let $E_{\varepsilon}^{(1)}$ be defined by (3.4). There holds:

(i) Let $\varepsilon_{k} \rightarrow 0$ and let $\left(u_{k}, \mu_{k}\right) \in L^{1}(\Omega) \times m_{+}(\Omega)$ be such that

$$
\sup _{k} E_{\varepsilon_{k}}^{(1)}\left(u_{k}, \mu_{k}\right)<+\infty .
$$

Then there exist a subsequence (not relabeled) such that $\left(u_{k}, \mu_{k}\right) \rightarrow(u, \mu)$ with respect to the $\tau_{1} \times \tau_{2}$ topology, for some $(u, \mu) \in L^{1}(\Omega) \times m_{+}(\Omega)$.

(ii) the family $\left(E_{\varepsilon}^{(1)}\right) \Gamma$-converges with respect to the $\tau_{1} \times \tau_{2}$ topology to the functional $E^{(1)}$ : $L^{1}(\Omega) \times m_{+}(\Omega) \rightarrow[0,+\infty]$ defined by

$$
E^{(1)}(u, \mu)= \begin{cases}\int_{S(u)} \varphi\left(\frac{d \mu}{d \mathcal{H}^{1}\lfloor S(u)}, v_{u}\right) d \mathcal{H}^{1}+(2 k-2)\left|\mu^{s}\right|(\Omega) & \text { if } u \in B V(\Omega ;\{ \pm 1\}), \\ +\infty & \text { otherwise }\end{cases}
$$

where, for $\mu \in \mathfrak{M}_{+}(\Omega)$, we have set $\mu^{s}:=\mu-\frac{d \mu}{d \mathfrak{H}^{1}\lfloor S(u)} \mathcal{H}^{1}\lfloor S(u)$ and the function $\varphi$ : $\mathbf{R}_{+} \times S^{1} \rightarrow[0,+\infty)$ is given by

$$
\varphi(z, v)=\max \left\{\varphi_{1}(z, v), \varphi_{2}(z, v), \varphi_{3}(z, v)\right\},
$$

where

$$
\begin{aligned}
& \varphi_{1}(z, v)=-4 k z+2\left(\left|v_{1}\right|+\left|v_{2}\right|\right), \\
& \varphi_{2}(z, v)=(1-3 k) z+2\left(\left|v_{1}\right| \vee\left|v_{2}\right|\right)+(1-k)\left(\left|v_{1}\right| \wedge\left|v_{2}\right|\right), \\
& \varphi_{3}(z, v)=2(1-k) z+(1-k)\left(\left|v_{1}\right|+\left|v_{2}\right|\right) .
\end{aligned}
$$

We postpone the proof of the previous theorem to Section 5, since it makes use of the integral representation result stated in Theorem 4.4. 


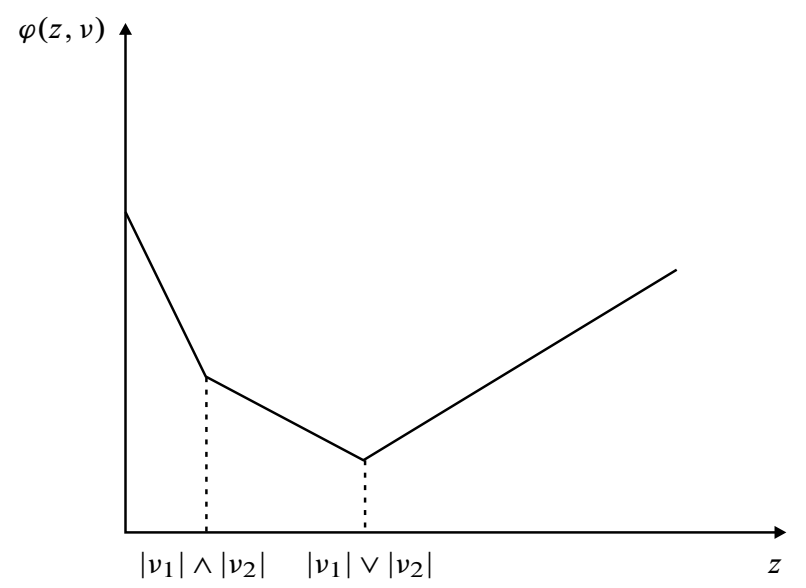

FIG. 3. The graph of the surface tension density $\varphi(z, v)$ as a function of the density $z$ of surfactant at the phase interface

REMARK 3.4 As it is clear from the graph of $\varphi$ (see Figure 2), a threshold phenomenon depending on the $v$ occurs at the phase interface. For a given $v \in S^{1}$ the surface tension $\varphi(z, v)$ of the system decreases only up to a certain value of the density $z$ of the surfactant, namely $z=\left|v_{1}\right| \vee\left|v_{2}\right|$. Increasing further the amount of surfactant in the system, the energy increases in two different ways. Either the density of the surfactant on the interfaces increases (the surfactant is absorbed onto the interface) and thus the surface tension grows, or the singular part of the surfactant measure increases (the surfactant is not absorbed onto the interface) and the energy grows proportionally to its mass.

As an application of the previous result, one may study the asymptotic behavior, as $\varepsilon \rightarrow 0$, of the following constrained optimization problem:

$$
m_{\varepsilon}:=\left\{E_{\varepsilon}^{(1)}(u), \varepsilon \# I_{0}(u)=\alpha_{\varepsilon}, \varepsilon^{2} \# I_{1}(u)=\beta_{\varepsilon}\right\}
$$

where

$$
I_{1}(u)=\left\{a \in \Omega_{\varepsilon}: u(a)=1\right\},
$$

$\lim _{\varepsilon} \alpha_{\varepsilon}=\alpha>0$ and $\lim _{\varepsilon} \beta_{\varepsilon}=\beta>0$. Since we are not interested in boundary layer effects, we consider the case when $\Omega$ is a torus, that we may identify with the semi-open cube $Q:=[0,1)^{2}$, $\varepsilon=\frac{1}{k}, k \in \mathbf{N}$, and the admissible functions $u$ in (3.7) are $Q$-periodic. The solution to this problem is a particular case of the result stated in Corollary 4.12 (see Remark 4.13).

\section{More general models}

In this section we consider a class of energies that generalizes those involved in the BEG model and in which long range interactions and different types of surfactant are taken into account.

Let $\Omega \subset \mathbf{R}^{n}$ be a bounded open set with Lipschitz boundary. We consider the family of functionals $F_{\varepsilon}: Q_{\varepsilon}(\Omega ; K) \rightarrow[0,+\infty)$ defined by

$$
F_{\varepsilon}(u)=\sum_{a, b \in \Omega_{\varepsilon},|a-b| \leqslant R \varepsilon} \varepsilon^{n-1} f\left(\frac{b-a}{\varepsilon}, u(a), u(b)\right),
$$


where $R>1, K=\left\{m_{1}, m_{2}, s_{1}, s_{2}, \ldots, s_{M}\right\} \subset \mathbf{R}, M \in \mathbf{N}$, and $f: \mathbf{Z}^{n} \times K^{2} \rightarrow[0,+\infty)$ satisfies the following hypotheses:

$$
\begin{gathered}
f^{-1}(0)=\mathbf{Z}^{n} \times\left\{\left(m_{1}, m_{1}\right),\left(m_{2}, m_{2}\right)\right\}, \\
f\left(\mathbb{R}^{i} \xi, u, v\right)=f(\xi, u, v) \quad \forall i \in\{1,2, \ldots, n\},
\end{gathered}
$$

where $R^{i}\left(\xi_{1}, \xi_{2}, \ldots, \xi_{i}, \ldots, \xi_{n}\right)=\left(\xi_{1}, \xi_{2}, \ldots,-\xi_{i}, \ldots, \xi_{n}\right)$ is the reflection with respect to the $i$-th coordinate axis. We also define a localized energy for every $A \subset \Omega$ as

$$
F_{\varepsilon}(u, A)=\sum_{a, b \in A \cap \varepsilon \mathbf{Z}^{n},|a-b| \leqslant R \varepsilon} \varepsilon^{n-1} f\left(\frac{b-a}{\varepsilon}, u(a), u(b)\right) .
$$

REMARK 4.1 We remark that (4.2) implies that the pure phases $u \equiv m_{i}, i=1,2$, are the ground states of the energy $F_{\varepsilon}$. Hypothesis (4.3) is a sort of discrete isotropy condition of the energy density and it is in particular satisfied if $f(\xi, u, v)=f(|\xi|, u, v)$.

REMARK 4.2 We observe that the functional $E_{\varepsilon}^{(1)}$ defined in (3.2) is of the type (4.1) with

$$
f(\xi, u, v):= \begin{cases}-u v-k(1-u v)^{2} & \text { if } \xi= \pm e_{i}, i \in\{1,2\}, \\ 0 & \text { otherwise, }\end{cases}
$$

which satisfies assumptions (4.2) and (4.3) with $K=\{ \pm 1,0\}, m_{1}=-1$ and $m_{2}=1$.

We now introduce the sets of points in $\Omega_{\varepsilon}$ occupied by the different types of surfactant and suitable measures associated to them. For $l \in\{1,2, \ldots, M\}$ we set, for all $A \subset \Omega$,

$$
\begin{gathered}
I_{l}(u, A)=\left\{a \in A \cap \Omega_{\varepsilon}: u(a)=s_{l}\right\}, \\
I(u, A)=\bigcup_{l=1}^{M} I_{l}(u, A)
\end{gathered}
$$

and, for the sake of simplicity, we set $I_{l}(u, \Omega)=I_{l}(u)$ and $I(u, \Omega)=I(u)$. Moreover we define

$$
\begin{gathered}
\mu_{l}(u):=\sum_{a \in I_{l}(u)} \varepsilon^{n-1} \delta_{a}, \\
\mu(u):=\left\{\mu_{1}(u), \mu_{2}(u), \ldots, \mu_{M}(u)\right\} .
\end{gathered}
$$

With the identification given in Remark 2.1, and a slight abuse of notation, we can extend $F_{\varepsilon}$ to a functional $F_{\varepsilon}: L^{1}(\Omega) \times\left(m_{+}(\Omega)\right)^{M} \rightarrow[0,+\infty]$ as

$$
F_{\varepsilon}(u, \mu):= \begin{cases}F_{\varepsilon}(u) & \text { if } u \in Q_{\varepsilon}(\Omega ; K), \mu=\mu(u), \\ +\infty & \text { otherwise. }\end{cases}
$$

We endow the space $L^{1}(\Omega) \times\left(m_{+}(\Omega)\right)^{M}$ with the topology $\tau_{1} \times \tau_{2}$ where $\tau_{1}$ denotes the strong topology in $L^{1}(\Omega)$ and $\tau_{2}$ denotes the weak ${ }^{*}$-topology in $\left(m_{+}(\Omega)\right)^{M}$. The choice of this topology is suggested by the following compactness result. 
Proposition 4.3 Let $\varepsilon_{k} \rightarrow 0$ and let $\left(u_{k}, \mu_{k}\right)$ be such that

$$
\sup _{k} F_{\varepsilon_{k}}\left(u_{k}, \mu_{k}\right)<+\infty .
$$

Then there exists a subsequence (not relabeled) such that $\left(u_{k}, \mu_{k}\right) \rightarrow(u, \mu)$ w.r.t. the $\tau_{1} \times \tau_{2^{-}}$ topology, for some $(u, \mu) \in B V\left(\Omega ;\left\{m_{1}, m_{2}\right\}\right) \times\left(m_{+}(\Omega)\right)^{M}$.

Proof. First note that

$$
\mathcal{H}^{n-1}\left(S\left(u_{k}\right)\right)+\mu_{k}(\Omega) \leqslant C F_{\varepsilon_{k}}\left(u_{k}, \mu_{k}\right) .
$$

By Theorem 2.2 and observing that

$$
\left|\left\{x \in \Omega: u_{k}(x) \notin\left\{m_{1}, m_{2}\right\}\right\}\right| \leqslant C \varepsilon_{k} \mu_{k}(\Omega) \rightarrow 0,
$$

one easily gets the conclusion.

\subsection{The main result}

In this section we state and prove an integral representation result for the $\Gamma$-limit of the family $F_{\varepsilon}$. To this end we introduce, for any $\varepsilon>0$ and $v \in S^{n-1}$, the class of discrete functions

$$
\mathrm{B}_{\varepsilon}\left(T Q_{\nu} ; K\right):=\left\{u \in \mathrm{Q}_{\varepsilon}\left(T Q_{\nu} ; K\right): u(a)=m_{1} \forall a \in \partial_{\varepsilon}^{+}\left(T Q_{\nu}\right), u(a)=m_{2} \forall a \in \partial_{\varepsilon}^{-}\left(T Q_{\nu}\right)\right\} .
$$

THEOREM 4.4 The family $\left(F_{\varepsilon}\right) \Gamma$-converges with respect to the $\tau_{1} \times \tau_{2}$-topology to the functional $F: L^{1}(\Omega) \times\left(m_{+}(\Omega)\right)^{M} \rightarrow[0,+\infty]$ defined by

$$
F(u, \mu)= \begin{cases}\int_{S(u)} f_{h o m}\left(\frac{d \mu}{d \mathfrak{H}^{n-1}\lfloor S(u)}, v(u)\right) d \mathcal{H}^{n-1}+\int_{\Omega} g_{h o m}\left(\mu^{s}\right) & \text { if } u \in B V\left(\Omega ;\left\{m_{1}, m_{2}\right\}\right), \\ +\infty & \text { otherwise, }\end{cases}
$$

where, for $\mu \in\left(\mathfrak{M}_{+}(\Omega)\right)^{M}$, we have set $\mu^{s}:=\mu-\frac{d \mu}{d \mathcal{H}^{n-1}\lfloor S(u)} \mathcal{H}^{n-1}\left\lfloor S(u)\right.$. Here, $f_{\text {hom }}:\left(\mathbf{R}_{+}\right)^{M} \times$ $S^{n-1} \rightarrow[0,,+\infty)$ is defined as

$$
\begin{array}{r}
f_{\text {hom }}(z, v):=\lim _{\delta \rightarrow 0^{+}} \lim _{T \rightarrow+\infty} \frac{1}{T^{n-1}} \inf \left\{F_{1}\left(u, T Q_{\nu}\right): u \in B_{1}\left(T Q_{\nu} ; K\right),\right. \\
\left.\max _{l \in\{1, \ldots, M\}}\left|\frac{\# I_{l}\left(u, T Q_{\nu}\right)}{T^{n-1}}-z_{l}\right|<\delta\right\},
\end{array}
$$

while $g_{\text {hom }}:\left(\mathbf{R}_{+}\right)^{M} \rightarrow[0,+\infty)$ is 1 -homogeneous and, for any $\zeta \in\left(\mathbf{R}_{+}\right)^{M}$ such that $\|\zeta\|_{1}=1$, is defined as

$$
\begin{aligned}
g_{\text {hom }}(\zeta):= & \lim _{\delta \rightarrow 0^{+}} \liminf _{T \rightarrow+\infty} \inf \left\{\frac{F_{1}(u, T Q)}{\# I(u, T Q)}: u \in Q_{1}(T Q ; K),\right. \\
& \left.\frac{F_{1}(u, T Q \backslash(T-R) Q)}{\# I(u, T Q)}<\delta, \max _{l \in\{1, \ldots, M\}}\left|\frac{\# I_{l}(u, T Q)}{\# I(u, T Q)}-\zeta_{l}\right|<\delta\right\} .
\end{aligned}
$$


REMARK 4.5 We observe that, while the formula for $f_{\text {hom }}$ can be proved by using standard arguments in homogenization theory, the same does not hold for $g_{\text {hom }}$. In particular, as it will be clear in the proof of Theorem 4.4, optimizing sequences for $f_{\text {hom }}(z, v)$ can be constructed, as it is usual in this framework, by "periodically gluing" a solution of the minimum problem on $T Q_{\nu}$ given in (4.9). In such a construction, the energy due to the interactions which cross the boundary of the periodicity cell is asymptotically negligible thanks to the Dirichlet type condition we are allowed to impose by using a De Giorgi's cut-off construction (see Lemma 4.9). The same arguments do not apply to $g_{\text {hom }}$. In fact, for this term, we cannot be sure that, imposing the same type of boundary conditions, we do not modify too much the energy of minimal configurations in (4.10) since the distribution of the phases $m_{1}$ and $m_{2}$ for such configurations is not known. This fact rules out the standard "periodic gluing" construction. Instead, we first make use of an abstract argument from measure theory which allows us to prove that the minimal configurations do not concentrate energy at the boundary of the periodic cell and then, by exploiting hypothesis (4.3), we construct optimizing sequences for $g_{\text {hom }}(\zeta)$ by a reflection argument (see the proof of Proposition 4.10).

Before proving Theorem 4.4 we show some properties enjoyed by $f_{h o m}$ and $g_{h o m}$. In the next Proposition we prove that the homogenization formula defining $f_{\text {hom }}$ is well defined.

Proposition 4.6 For any $z \in \mathbf{R}^{M}, v \in S^{n-1}$ and $\delta>0$ there exists the limit

$$
\lim _{T \rightarrow+\infty} \frac{1}{T^{n-1}} \inf \left\{F_{1}\left(u, T Q_{v}\right): u \in B_{1}\left(T Q_{v} ; K\right), \max _{l \in\{1, \ldots, M\}}\left|\frac{\# I_{l}\left(u, T Q_{v}\right)}{T^{n-1}}-z_{l}\right|<\delta\right\} .
$$

Proof. Let us fix $v \in S^{n-1}$. For $k \in\{1, \ldots, n\}$ we label the $(n-1)$-dimensional faces of $Q_{v}$ as $\left\{F_{k}^{-}, F_{k}^{+}\right\}$. They are given by

$$
F_{k}^{ \pm}=\bar{Q}_{v} \cap\left\{x_{k}= \pm r_{v}\right\}
$$

Moreover we set $P_{k}^{ \pm}=\Pi_{\nu} \cap F_{k}^{ \pm}$and observe that, by symmetry, for any $k \in\{1, \ldots, n\}$ either $P_{k}^{-}=P_{k}^{+}=\emptyset$ or there exist $y^{k} \in \mathbf{R}^{n}$ such that $P_{k}^{+}=P_{k}^{-}+y^{k}$. Note that, set $J=\left\{k: P_{k}^{-}=\right.$ $\left.P_{k}^{+}=\emptyset\right\}$, we have that $\# J \leqslant 1$ and that $\left\{y^{k}\right\}_{k \notin J}$ have rank $(n-1)$ and span $\Pi_{\nu}$. Moreover, since by construction $y_{k}^{k}=2 r_{v}\left(y_{k}^{k}\right.$ is the $k$-th component of $\left.y^{k}\right)$, then, set $\hat{Q}_{\nu}=\left[-r_{v}, r_{v}\right)^{n}$, the cubes in $Q:=\left\{\hat{Q}_{\nu}+\sum_{k \notin J} m_{k} y^{k}: m_{k} \in \mathbf{Z}\right\}$ are pairwise disjoint and cover $\Pi_{\nu}$.

Let us define

$$
I_{T}(z, \delta):=\inf \left\{F_{1}\left(u, T Q_{v}\right): u \in B_{1}\left(T Q_{v} ; K\right), \max _{l \in\{1, \ldots, M\}}\left|\frac{\# I_{l}\left(u, T Q_{\nu}\right)}{T^{n-1}}-z_{l}\right|<\delta\right\},
$$

and, given $\eta>0$, let $u_{T} \in \mathbb{B}_{1}\left(T Q_{\nu} ; K\right)$ be such that $\max _{l \in\{1, \ldots, M\}}\left|\frac{\# I_{l}\left(u, T Q_{\nu}\right)}{T^{n-1}}-z_{l}\right|<\delta$ and

$$
F_{1}\left(u_{T}, T Q_{v}\right) \leqslant I_{T}(z, \delta)+\eta .
$$

Extend $u_{T}$ on $Q_{v}^{T}:=\left\{x \in \mathbf{R}^{n}: \operatorname{dist}\left(x, T Q_{v}\right) \leqslant \sqrt{n}\right\}$ by setting for $a \in \mathbf{Z}^{n} \cap\left(Q_{v}^{T} \backslash T Q_{v}\right)$

$$
u_{T}(a)=m_{1} \text { if }(a, v) \geqslant 0 \quad u_{T}(a)=m_{2} \text { if }(a, v) \leqslant 0 .
$$

Given $S>T$, set $Q_{S}:=\left\{Q \in T Q: Q \subset S Q_{\nu}\right.$ and $\left.Q \cap \Pi_{\nu} \neq \emptyset\right\}$ and let $v_{S} \in \mathbb{B}_{1}\left(S Q_{\nu} ; K\right)$ be defined as follows. Let $\bar{y}:=T \sum_{k \notin J} m_{k} y^{k}, m_{k} \in \mathbf{Z}$, be such that $T \hat{Q}_{v}+\bar{y} \in Q_{S}$. Then set

$$
v_{S}(a)=u_{T}(a-[\bar{y}]), \text { for } a \in \mathbf{Z}^{n} \cap\left(T \hat{Q}_{v}+\bar{y}\right),
$$



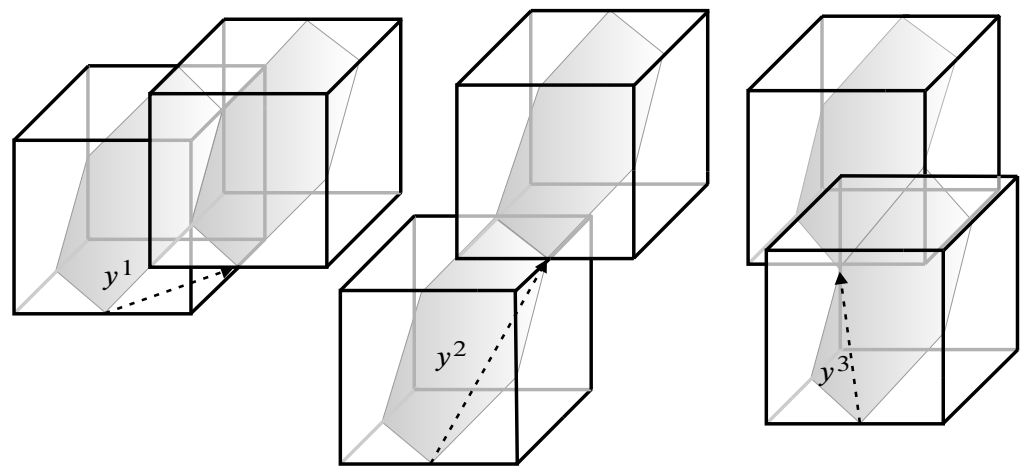

FIG. 4. The translation vectors $\left\{y^{1}, y^{2}, y^{3}\right\}$ in Proposition 4.6 in the case $n=3$ and $J=\emptyset$. The sets $Q_{v} \cap \Pi_{v}$ and $y^{i}+Q_{v} \cap \Pi_{v}$ for $i=1,2,3$ are shaded.

where by $[\bar{y}]$ we denote the point in $\mathbf{R}^{n}$ whose components are the integer part of the components of $\bar{y}$. For the remaining $a \in \mathbf{Z}^{n} \cap\left(S Q_{v} \backslash \bigcup_{Q \in Q_{S}} Q\right)$, set

$$
v_{S}(a)=m_{1} \text { if }(a, v) \geqslant 0 \quad u_{S}(a)=m_{2} \text { if }(a, v) \leqslant 0 .
$$

Note that, for $S$ large enough, we have

$$
\left|\frac{\# I_{l}\left(v_{S}, S Q\right)}{S^{n-1}}-z_{l}\right|<\delta .
$$

Hence, splitting the energy into two terms, the first one accounting for the interactions inside each cube in $Q_{S}$ and the second one accounting for the interactions which cross the boundary of the same cubes and those in $S Q_{\nu} \backslash \bigcup_{Q \in Q_{S}} Q$, we get

$$
\begin{aligned}
\frac{1}{S^{n-1}} I_{S}(z, \delta) \leqslant \frac{1}{S^{n-1}} F_{1}\left(v_{S}, S Q_{\nu}\right) & \leqslant \frac{1}{S^{n-1}}\left[\frac{S^{n-1}}{T^{n-1}}\right]\left(F_{1}\left(u_{T}, T Q_{\nu}\right)+C T^{n-2}\right) \\
& \leqslant \frac{T^{n-1}}{S^{n-1}}\left[\frac{S^{n-1}}{T^{n-1}}\right]\left(\frac{1}{T^{n-1}} I_{T}(z, \delta)+\frac{C T^{n-2}+\eta}{T^{n-1}}\right) .
\end{aligned}
$$

By letting first $S$ and then $T$ go to $+\infty$, by the arbitrariness of $\eta$ we finally get

$$
\limsup _{S \rightarrow+\infty} \frac{1}{S^{n-1}} I_{S}(z, \delta) \leqslant \liminf _{T \rightarrow+\infty} \frac{1}{T^{n-1}} I_{T}(z, \delta) .
$$

In the next proposition we prove growth and convexity properties of the functions $f_{\text {hom }}$ and $g_{\text {hom }}$. We remark that, in the proof the $\Gamma$-convergence result, we only use the continuity of $f_{\text {hom }}$ and $g_{\text {hom }}$ and that their convexity would be a consequence of the lower semicontinuity of the $\Gamma$-limit. However the proof of the continuity would rely on the same argument we exploit here without providing any significant simplification.

Proposition 4.7 Let $f_{\text {hom }}$ and $g_{\text {hom }}$ be defined as in (4.9) and (4.10). There holds 
(i) the 1-homogeneous extension of $f_{\text {hom }}$ in $\left(\mathbf{R}_{+}\right)^{M} \times \mathbf{R}^{n}$ is convex. Moreover there exists $C>0$ such that

$$
f_{\text {hom }}(z, v) \leqslant C(|z|+1), \quad \text { for all }(z, v) \in\left(\mathbf{R}_{+}\right)^{M} \times S^{n-1},
$$

(ii) $g_{h o m}$ is convex.

Proof. Proof of (i)

By the 1-homogeneity of $f_{\text {hom }}$ the proof of its convexity reduces to prove that, given $v, v^{(1)}, v^{(2)} \in$ $S^{n-1}$ and $z, z^{(1)}, z^{(2)} \in\left(\mathbf{R}_{+}\right)^{M}$ such that $(z, v)=l_{1}\left(z^{(1)}, v^{(1)}\right)+l_{2}\left(z^{(2)}, v^{(2)}\right)$, for $l_{1}, l_{2} \in \mathbf{R}$, there holds

$$
f_{\text {hom }}(z, v) \leqslant l_{1} f_{\text {hom }}\left(z^{(1)}, v^{(1)}\right)+l_{2} f_{\text {hom }}\left(z^{(2)}, v^{(2)}\right) .
$$

The idea of the proof of (4.13) is the following: given $\eta>0$, let $\delta>0, T>\frac{1}{\eta}$ and let $u_{1} \in$ $\mathrm{B}_{1}\left(T Q_{v^{(1)}} ; K\right), u_{2} \in \mathrm{B}_{1}\left(T Q_{v^{(2)}} ; K\right)$ such that, for $i \in\{1,2\}$ it holds

$$
\begin{array}{r}
\frac{1}{T^{n-1}} F_{1}\left(u_{i}, T Q_{v^{(i)}}\right) \leqslant f_{\text {hom }}\left(z^{(i)}, v^{(i)}\right)+\eta, \\
\max _{l \in\{1, \ldots, M\}}\left|\frac{\# I_{l}\left(u_{i}, T Q_{v^{(i)}}\right)}{T^{n-1}}-z_{l}^{(i)}\right|<\delta .
\end{array}
$$

Then for $S>>T$ one uses a zig-zag construction to define a test function $u_{S} \in B_{1}\left(S Q_{\nu} ; K\right)$ such that

$$
\frac{1}{S^{n-1}} F_{1}\left(u_{S}, S Q_{\nu}\right) \leqslant \sum_{i=1}^{2} c_{i}(T, S) \frac{1}{T^{n-1}} F_{1}\left(u_{i}, T Q_{v^{(i)}}\right)+R(T, S)+o(\eta),
$$

with $\lim _{S \rightarrow+\infty} \lim _{T \rightarrow+\infty} R(T, S)=0$ and $\lim _{S \rightarrow+\infty} \lim _{T \rightarrow+\infty} c_{i}(T, S)=l_{i}, i=1,2$. The inequality (4.13) follows from this last estimate by the arbitrariness of $\eta>0$.

We will provide a detailed construction of $u_{S}$ under the additional assumption that $l_{1}, l_{2}>0$, $v=e_{n}, v^{(1)}, v^{(2)} \in\left\{x \in \mathbf{R}^{n}: x_{1}=\cdots=x_{n-2}=0\right\}=: \Pi,\left(v^{(1)}, v^{(2)}\right) \geqslant 0$ and the ordered base $\left\{v^{(1)}, v^{(2)}\right\}$ has the same orientation as $\left\{e_{n}, e_{n-1}\right\}$. In the general case the construction is similar and would only require an extra amount of notation without adding new insight in the understanding of the proof.

Given $\eta>0, \delta>0, T>\frac{1}{\eta}$, let then $u_{1} \in \mathbb{B}_{1}\left(T Q_{v^{(1)}} ; K\right), u_{2} \in \mathbb{B}_{1}\left(T Q_{v^{(2)}} ; K\right)$ satisfying (4.14) for $i \in\{1,2\}$. Let us set, for $i \in\{1,2\}, r_{i}:=2 r_{v^{(i)}}$, so that $Q_{v^{(i)}}=\left(-\frac{r_{i}}{2}, \frac{r_{i}}{2}\right)^{n}$, and let us consider $u_{i}$ identified with its piecewise-constant interpolation and extended to $\mathbf{R}^{n-2} \times T\left[-\frac{r_{i}}{2}, \frac{r_{i}}{2}\right]^{2}$ by periodicity in the $\left\{e_{1}, \ldots, e_{n-2}\right\}$ directions. Moreover we set $\left(v^{(i)}\right)^{\perp}=\left(0, \ldots, 0, v_{n}^{(i)},-v_{n-1}^{(i)}\right)$ and $L_{i}:=H^{1}\left(\left\{x=t\left(v^{(i)}\right)^{\perp}, t \in \mathbf{R}\right\} \cap Q_{v^{(i)}}\right)$. We can then further extend $u_{i}$ by periodicity in the direction $\left(v^{(i)}\right)^{\perp}$ without renaming it, that is

$$
u_{i}\left(x+L_{i}\left(v^{(i)}\right)^{\perp}\right)=u_{i}(x), \quad x \in \mathbf{R}^{n-2} \times \bigcup_{j \in \mathbf{Z}}\left(T\left[-\frac{r_{i}}{2}, \frac{r_{i}}{2}\right]^{2}+j L_{i}\left(v^{(i)}\right)^{\perp}\right) .
$$

We now use a zig-zag construction to define a good test function $u_{S} \in B_{1}(S Q ; K)$, for $S>>T$, in the minimum problem occurring in the definition of $f_{\text {hom }}(z, v)$ (see Figure 3). Let us set $V:=$ $\left\{x \in \mathbf{R}^{n}: 0 \leqslant x_{n-1} \leqslant 1\right\}, \Pi_{v}^{ \pm}:=\left\{x \in \mathbf{R}^{n}: \pm(x, v) \geqslant 0\right\}, v \in S^{n-1}$, and let $w: V \rightarrow\left\{m_{1}, m_{2}\right\}$ be defined as

$$
w(x)= \begin{cases}m_{1} & \text { if } x \in \Pi_{v^{(1)}}^{+} \cup\left(\Pi_{v^{(2)}}^{+}+l_{1}\left(v^{(1)}\right)^{\perp}\right), \\ m_{2} & \text { otherwise. }\end{cases}
$$



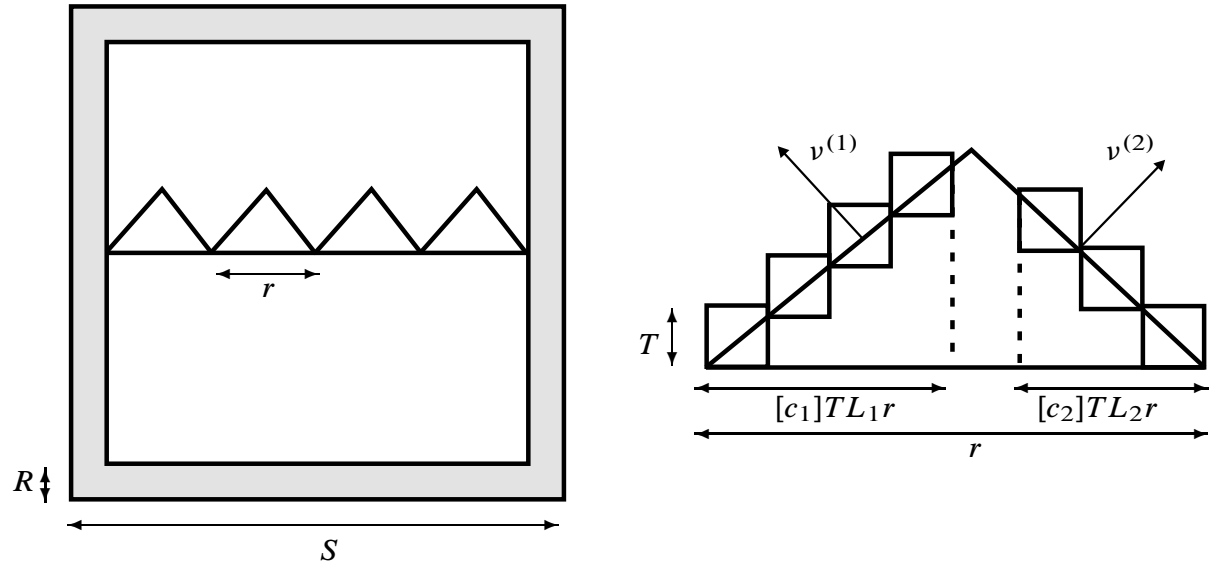

FIG. 5. The zig-zag construction leading to $u_{S}$ in the proof of Proposition 4.7. On the right a zoom of one of the triangles on the left.

Let $c_{1}, c_{2}>0$ be such that $c_{1} \frac{T L_{1}}{l_{1}}=c_{2} \frac{T L_{2}}{l_{2}}=: r$. Let $u: \mathbf{R}^{n} \rightarrow \mathbf{R}$ be the $r e_{n-1}$-periodic function defined in $r V$ as

$$
u(x)= \begin{cases}u_{1}(x) & \text { if } 0 \leqslant x_{n-1} \leqslant\left[c_{1}\right] T L_{1}\left(v^{(1)}, e_{n}\right), \\ u_{2}\left(x-r e_{n-1}\right) & \text { if } r-\left[c_{2}\right] T L_{2}\left(v^{(2)}, e_{n}\right) \leqslant x_{n-1} \leqslant r \\ w\left(\frac{x}{r}\right) & \text { otherwise in } r V .\end{cases}
$$

and periodically extended in the direction $e_{n-1}$. Let $S>>T$, we define $u_{S} \in \mathbb{B}_{1}\left(S Q_{\nu} ; K\right)$ such that

$$
u_{S}(a)= \begin{cases}u(a) & \text { if } a \in(S-R) Q_{v} \cap \mathbf{Z}^{n}, \\ u_{v}(a) & \text { otherwise in } S Q_{v} \cap \mathbf{Z}^{n} .\end{cases}
$$

We can now estimate the energy of $u_{S}$ :

$\frac{1}{S^{n-1}} F_{1}\left(u_{S}, S Q_{\nu}\right) \leqslant \frac{1}{S^{n-1}} \sum_{i=1}^{2}\left[\frac{S}{r}\right]\left[c_{i}\right]\left[\frac{S}{T r_{i}}\right]^{n-2}\left(F_{1}\left(u_{i}, T Q_{\nu^{(i)}}\right)+C T^{n-2}\right)+\frac{1}{S^{n-1}} C S^{n-2}$,

where the term of the type $C T^{n-2}$ is the energetic contribution due to the interactions near each set of the type $\partial\left(T Q_{v^{(i)}}\right) \cap \Pi_{v^{(i)}}$, while the term of the type $C S^{n-2}$ is due to the interactions near $\partial\left(S Q_{v}\right) \cap \Pi_{v}$. By construction we have that, for $l \in\{1, \ldots, M\}$,

$$
\frac{\# I_{l}\left(u_{S}, S Q_{\nu}\right)}{S^{n-1}}=\frac{1}{S^{n-1}} \sum_{i=1}^{2}\left[\frac{S}{r}\right]\left[c_{i}\right]\left[\frac{S}{T r_{i}}\right]^{n-2} \# I_{l}\left(u_{i}, T Q_{v^{(i)}}\right)+o(1) .
$$

Taking into account the definition of $c_{i}$ and the fact that by construction $r_{i}^{n-2} L_{i}=1$ we have that, for $T$ and $S$ large enough,

$$
\frac{1}{S^{n-1}}\left[\frac{S}{r}\right]\left[c_{i}\right]\left[\frac{S}{T r_{i}}\right]^{n-2} \leqslant \frac{l_{1}+C \eta}{T^{n-1}}
$$


Then, by (4.16), for $T$ and $S$ large enough $u_{S}$ is a good test function in the minimum problem defining $f_{\text {hom }}(z, v)$ and, by (4.15) we get

$$
f_{\text {hom }}(z, v) \leqslant l_{1} f_{\text {hom }}\left(z^{(1)}, v^{(1)}\right)+l_{2} f_{\text {hom }}\left(z^{(2)}, v^{(2)}\right)+C \eta
$$

which eventually gives (4.13) by the arbitrariness of $\eta$.

To obtain the estimate (4.12) we observe that we can rewrite the energy $F_{1}\left(u, T Q_{v}\right)$ as

$$
F_{1}\left(u, T Q_{v}\right)=\sum_{(a, b) \in D(u)} f(b-a, u(a), u(b))+F_{1}^{s u r}\left(u, T Q_{v}\right)
$$

where

$$
D(u):=\left\{(a, b) \in\left(\mathbf{Z}^{n} \cap T Q_{\nu}\right)^{2}: 0<|b-a| \leqslant R, u(a) \neq u(b),\{u(a), u(b)\}=\left\{m_{1} m_{2}\right\}\right\} .
$$

Here $F_{1}^{\text {sur }}$ is the energy accounting only for the contribution due to the interactions of surfactant type particles. Note that, since $f$ is bounded, the first term in the right hand side of (4.18) is proportional to $\# D(u)$ and, since each particle has only an equi-bounded number of interactions, $F_{1}^{s u r}\left(u, T Q_{v}\right)$ is proportional to \#I $\left(u, T Q_{v}\right)$. Then the estimate (4.12) is achieved by choosing, in the problem defining $f_{\text {hom }}(z, v)$ any test function $u$ such that $\# D(u) \simeq C T^{n-1}$.

Proof of (ii). In order to prove (ii), by the 1-homogeneity of $g_{\text {hom }}$, it is enough to show that, given $\zeta^{(1)}, \zeta^{(2)} \in\left(\mathbf{R}_{+}\right)^{M}$ with $\left\|\zeta^{(1)}\right\|_{1}=\left\|\zeta^{(2)}\right\|_{1}=1$ and $t \in(0,1)$ it holds that

$$
g_{\text {hom }}\left(t \zeta^{(1)}+(1-t) \zeta^{(2)}\right) \leqslant t g_{h o m}\left(\zeta^{(1)}\right)+(1-t) g_{h o m}\left(\zeta^{(2)}\right) .
$$

For any $\delta>0$ and $\zeta \in\left(\mathbf{R}_{+}\right)^{M}$ with $\|\zeta\|_{1}=1$, we set

$$
\begin{aligned}
g(\delta, \zeta):=\liminf _{T \rightarrow+\infty} \inf & \left\{\frac{F_{1}(u, T Q)}{\# I(u, T Q)}: u \in Q_{1}(T Q ; K),\right. \\
& \left.\frac{F_{1}(u, T Q \backslash(T-R) Q)}{\# I(u, T Q)}<\delta, \max _{l \in\{1, \ldots, M\}}\left|\frac{\# I_{l}(u, T Q)}{\# I(u, T Q)}-\zeta_{l}\right|<\delta\right\} .
\end{aligned}
$$

Given $\delta>0$, for $i \in\{1,2\}$ let $u_{i} \in Q(T Q ; K)$ such that

$$
\begin{aligned}
& \frac{F_{1}\left(u_{i}, T Q\right)}{\# I\left(u_{i}, T Q\right)}<g\left(\delta, \zeta^{(i)}\right)+\delta, \\
& \frac{F_{1}\left(u_{i}, T Q \backslash(T-R) Q\right)}{\# I\left(u_{i}, T Q\right)}<\delta \\
& \max _{l \in\{1, \ldots, M\}}\left|\frac{\# I_{l}\left(u_{i}, T Q\right)}{\# I\left(u_{i}, T Q\right)}-\zeta_{l}^{(i)}\right|<\delta .
\end{aligned}
$$

Set $\tilde{\zeta}=t \zeta^{(1)}+(1-t) \zeta^{(2)}$, we now construct a suitable test function in the minimum problem defining $g(\delta, \tilde{\zeta})$. Let $S=k T$ with $1<<k \in \mathbf{N}$. Let $h \in \mathbf{N}, h<k$ be such that, for $k$ large enough, set

$$
\lambda(h):=\frac{h \# I\left(u_{1}, T Q\right)}{h \# I\left(u_{1}, T Q\right)+(k-h) \# I\left(u_{2}, T Q\right)},
$$

there holds

$$
|t-\lambda(h)|<\delta .
$$


Without loss of generality we choose $T$ to be an even number. We extend $u_{i}$ by reflection with respect to the coordinate axes. More precisely we set $u_{i, 0}=u_{i}$ and, for all $j \in\{1, \ldots, n\}$, we define $u_{i, j}$ recursively as follows: $u_{i, j}$ is the extension of $u_{i, j-1}$ on $\mathbf{R}^{j} \times\left[-\frac{T}{2}, \frac{T}{2}\right]^{n-j}$ satisfying the following property

$$
u_{i, j}(a)=u_{i, j}\left(a+\left(T-2 a_{j}\right) e_{j}\right), \quad \forall a \in \mathbf{R}^{j-1} \times\left[-\frac{T}{2}, \frac{T}{2}\right]^{n-(j-1)}+m T e_{j}, \quad m \in \mathbf{Z}
$$

We have then obtained that the function $u_{i, n}$ extends $u_{i}$ on all $\mathbf{Z}^{n}$. Let us observe that, by the symmetry hypotheses in (4.2), we have that

$$
F_{1}\left(u_{i, n}, T Q+m\right)=F_{1}\left(u_{i}, T Q\right) \quad \forall m \in \mathbf{Z}^{n}
$$

Let $u: \mathbf{Z}^{n} \cap S Q \rightarrow K$ be defined as

$$
u(a)= \begin{cases}u_{1, n}(a) & \text { if }-\frac{S}{2} \leqslant a_{n} \leqslant-\frac{S}{2}+h, \\ u_{2, n}(a) & \text { otherwise. }\end{cases}
$$

By (4.21) and (4.22) we have that

$$
\begin{aligned}
\left|\frac{\# I_{l}(u, S Q)}{\# I(u, S Q)}-\tilde{\zeta}_{l}\right| & =\left|\frac{k^{n-1} h \# I_{l}\left(u_{1}, T Q\right)+k^{n-1}(k-h) \# I_{l}\left(u_{2}, T Q\right)}{k^{n-1} h \# I\left(u_{1}, T Q\right)+k^{n-1}(k-h) \# I\left(u_{2}, T Q\right)}-\tilde{\zeta}_{l}\right| \\
& \leqslant \lambda(h)\left|\frac{\# I_{l}\left(u_{1}, T Q\right)}{\# I\left(u_{1}, T Q\right)}-\zeta_{l}^{(1)}\right|+(1-\lambda(h))\left|\frac{\# I_{l}\left(u_{2}, T Q\right)}{\# I\left(u_{2}, T Q\right)}-\zeta_{l}^{(2)}\right| \\
& +\left(\left\|\zeta^{(1)}\right\|_{1} \vee\left\|\zeta^{(2)}\right\|_{1}\right) \delta \\
& \leqslant\left(1+\left\|\zeta^{(1)}\right\|_{1} \vee\left\|\zeta^{(2)}\right\|_{1}\right) \delta .
\end{aligned}
$$

We now estimate the energy $\frac{F_{1}(u, S Q)}{\# I(u, S Q)}$ :

$$
\frac{F_{1}(u, S Q)}{\# I(u, S Q)} \leqslant \frac{k^{n-1} h F_{1}\left(u_{1}, T Q\right)+k^{n-1}(k-h) F_{1}\left(u_{2}, T Q\right)}{\# I(u, S Q)}+R_{1}+R_{2}
$$

Here the first term in the right hand side is obtained by taking into account (4.23). Moreover $R_{1}$ is the energy due to the interactions which cross the set $S Q \cap\left\{x_{n}=-\frac{S}{2}+h\right\}$, where in the construction of $u$ we pass from $u_{1}$ to $u_{2}$, while $R_{2}$ accounts for all the other interactions which cross the boundary of the cubes of type $T Q+m$ with $m \in \mathbf{Z}$. An easy computation shows that

$$
R_{1} \leqslant C \frac{k^{n-1} T}{\# I(u, S Q)} \leqslant C \frac{T}{k}
$$


In addition, thanks to (4.21) we get

$$
\begin{aligned}
R_{2} & \leqslant \frac{k^{n-1} h F_{1}\left(u_{1}, T Q \backslash(T-R) Q\right)+k^{n-1}(k-h) F_{1}\left(u_{2}, T Q \backslash(T-R) Q\right)}{\# I(u, S Q)} \\
& =\frac{k^{n-1} h F_{1}\left(u_{1}, T Q \backslash(T-R) Q\right)+k^{n-1}(k-h) F_{1}\left(u_{2}, T Q \backslash(T-R) Q\right)}{k^{n-1} h \# I\left(u_{1}, T Q\right)+k^{n-1}(k-h) \# I\left(u_{2}, T Q\right)} \\
& \leqslant \lambda(h) \frac{F_{1}\left(u_{1}, T Q \backslash(T-R) Q\right)}{\# I\left(u_{1}, T Q\right)}+(1-\lambda(h)) \frac{F_{1}\left(u_{2}, T Q \backslash(T-R) Q\right)}{\# I\left(u_{2}, T Q\right)} \\
& \leqslant \delta .
\end{aligned}
$$

Hence, using the same argument to estimate the first term in the right hand side of (4.24), by (4.21), (4.22) and the estimates of $R_{1}$ and $R_{2}$, we have

$$
\begin{aligned}
\frac{F_{1}(u, S Q)}{\# I(u, S Q)} & =\lambda(h) \frac{F_{1}\left(u_{1}, T Q\right)}{\# I\left(u_{1}, T Q\right)}+(1-\lambda(h)) \frac{F_{1}\left(u_{2}, T Q\right)}{\# I\left(u_{2}, T Q\right)}+R_{1}+R_{2} \\
& \leqslant \operatorname{tg}\left(\delta, \zeta^{(1)}\right)+(1-t) g\left(\delta, \zeta^{(2)}\right)+C\left(\delta+\frac{T}{k}\right) .
\end{aligned}
$$

Letting $k$ go to $+\infty$ we get

$$
g(C \delta, \zeta) \leqslant \operatorname{tg}\left(\delta, \zeta^{(1)}\right)+(1-t) g\left(\delta, \zeta^{(2)}\right)+C \delta
$$

which eventually gives the conclusion letting $\delta$ go to 0 .

The proof of Theorem 4.4 is a consequence of Propositions 4.8 and 4.10 in which we prove the $\Gamma$-lim inf and the $\Gamma$-lim sup inequality, respectively.

Proposition 4.8 ( $\Gamma$-lim inf inequality) We have

$$
\Gamma-\liminf _{\varepsilon \rightarrow 0} F_{\varepsilon}(u, \mu) \geqslant F(u, \mu) .
$$

Proof. Let $\varepsilon_{k} \rightarrow 0$. Up to subsequences, it suffices to consider $\left(u_{k}, \mu_{k}\right) \rightarrow(u, \mu)$ w.r.t. the $\tau_{1} \times \tau_{2^{-}}$ topology such that

$$
\liminf _{k} F_{\varepsilon_{k}}\left(u_{k}, \mu_{k}\right)=\lim _{k} F_{\varepsilon_{k}}\left(u_{k}, \mu_{k}\right)<+\infty .
$$

By Proposition 4.3 we have that $u \in B V\left(\Omega ;\left\{m_{1}, m_{2}\right\}\right)$. We now consider the family of measures $\left(\lambda_{k}\right)_{k} \subset m_{+}(\Omega)$ defined as

$$
\lambda_{k}:=\sum_{a \in \Omega_{\varepsilon_{k}}} \sum_{b \in \Omega_{\varepsilon_{k}}:|a-b| \leqslant R \varepsilon_{k}} \varepsilon_{k}^{n-1} f\left(\frac{b-a}{\varepsilon_{k}}, u(a), u(b)\right) \delta_{a} .
$$

Note that $\lambda_{k}(\Omega)=F_{\varepsilon_{k}}\left(u_{k}, \mu_{k}\right)$. Then, by (4.26), we may suppose that, up to extracting a subsequence (not relabeled), there exist $\lambda \in m_{+}(\Omega)$ such that $\lambda_{k} \rightarrow \lambda$. We now use a blowup argument. By the Radon-Nikodym Theorem we may decompose $\mu$ into two mutually singular measures in $\left(m_{+}(\Omega)\right)^{M}$

$$
\mu=z \mathcal{H}^{n-1}\left\lfloor S(u)+\mu^{s}=z \mathcal{H}^{n-1}\left\lfloor S(u)+\zeta\left\|\mu^{s}\right\|_{1}\right.\right.
$$


and $\lambda$ into three mutually singular non-negative measures

$$
\lambda=\xi \mathcal{H}^{n-1}\left\lfloor S(u)+\eta\left\|\mu^{s}\right\|_{1}+\lambda^{s},\right.
$$

where $\left\|\mu^{s}\right\|_{1}:=\sum_{l=1}^{M} \mu_{l}^{s}$. Hence we complete the proof if we show that

$$
\xi\left(x_{0}\right) \geqslant f_{\text {hom }}\left(z\left(x_{0}\right), v_{u}\left(x_{0}\right)\right), \quad \text { for } \mathcal{H}^{n-1} \text {-a.e. } x_{0} \in S(u),
$$

and that

$$
\eta\left(x_{0}\right) \geqslant g_{\text {hom }}\left(\zeta\left(x_{0}\right)\right), \quad \text { for }\left|\mu^{s}\right|-\text { a.e. } x_{0} \in \Omega .
$$

The proof of (4.28) and (4.29) will be performed in two steps.

Step 1. Proof of (4.28).

By the properties of $B V$ functions (see [10]) we have that, for $\mathcal{H}^{n-1}$-a.e. $x_{0} \in S(u)$ it holds

(i) $\lim _{\rho \rightarrow 0^{+}} \frac{1}{\rho^{n}} \int_{x_{0}+\rho Q_{\nu_{u}\left(x_{0}\right)}^{ \pm}}\left|u(x)-u^{ \pm}\left(x_{0}\right)\right| d x=0$,

(ii) $\lim _{\rho \rightarrow 0^{+}} \frac{1}{\rho^{n-1}} \mathcal{H}^{n-1}\left(S(u) \cap\left\{x_{0}+\rho Q_{v_{u}\left(x_{0}\right)}\right\}\right)=1$,

(iii) $\xi\left(x_{0}\right)=\lim _{\rho \rightarrow 0^{+}} \frac{1}{\rho^{n-1}} \lambda\left(\left\{x_{0}+\rho Q_{v_{u}\left(x_{0}\right)}\right\}\right)$,

(iv) $z\left(x_{0}\right)=\lim _{\rho \rightarrow 0^{+}} \frac{1}{\rho^{n-1}} \mu\left(\left\{x_{0}+\rho Q_{v_{u}\left(x_{0}\right)}\right\}\right)$.

Fix such a $x_{0} \in S(u)$ and let $\left(\rho_{m}\right)$ be a sequence of positive numbers converging to zero such that

$$
\lambda\left(\partial\left\{x_{0}+\rho_{m} Q_{v_{u}\left(x_{0}\right)}\right\}\right)=0, \quad|\mu|\left(\partial\left\{x_{0}+\rho_{m} Q_{v_{u}\left(x_{0}\right)}\right\}\right)=0 .
$$

By (ii) and (iii) we get

$$
\begin{aligned}
\xi\left(x_{0}\right) & =\lim _{m} \frac{1}{\rho_{m}^{n-1}} \lambda\left(\left\{x_{0}+\rho_{m} Q_{v_{u}\left(x_{0}\right)}\right\}\right) \\
& \geqslant \lim _{m} \lim _{k} \frac{1}{\rho_{m}^{n-1}} F_{\varepsilon_{k}}\left(u_{k},\left\{x_{0}+\rho_{m} Q_{v_{u}\left(x_{0}\right)}\right\}\right),
\end{aligned}
$$

and by (iv) we have

$$
\lim _{m} \lim _{k} \frac{1}{\rho_{m}^{n-1}} \mu\left(u_{k}\right)\left(\left\{x_{0}+\rho_{m} Q_{v_{u}\left(x_{0}\right)}\right\}\right)=z\left(x_{0}\right) .
$$

Observe that, for every $m$ and $k$ we can find $\rho_{m, k}$ with $\lim _{k} \rho_{m, k}=\rho_{m}$ and $x_{0}^{k} \in \varepsilon_{k} \mathbf{Z}^{n}$ with $\lim _{k} x_{0}^{k}=x_{0}$, such that

$$
\varepsilon_{k} \mathbf{Z}^{n} \cap\left(\left\{x_{0}^{k}+\rho_{m, k} Q_{v_{u}\left(x_{0}\right)}\right\}\right)=\varepsilon_{k} \mathbf{Z}^{n} \cap\left\{x_{0}+\rho_{m} Q_{v_{u}\left(x_{0}\right)}\right\}
$$

which in turn implies

$$
F_{\varepsilon_{k}}\left(u_{k},\left\{x_{0}+\rho_{m} Q_{v_{u}\left(x_{0}\right)}\right\}\right)=F_{\varepsilon_{k}}\left(u_{k},\left\{x_{0}^{k}+\rho_{m, k} Q_{v_{u}\left(x_{0}\right)}\right\}\right) .
$$


Then

$$
\xi\left(x_{0}\right) \geqslant \lim _{m} \lim _{k} \frac{1}{\rho_{m, k}^{n-1}} F_{\varepsilon_{k}}\left(u_{k},\left\{x_{0}^{k}+\rho_{m, k} Q_{v_{u}\left(x_{0}\right)}\right\}\right) .
$$

Let us set

$$
u_{m, k}(a)=u_{k}\left(x_{0}^{k}+\rho_{m, k} a\right), \quad a \in \frac{\varepsilon_{k}}{\rho_{m, k}} \mathbf{Z}^{n} \cap Q_{v_{u}\left(x_{0}\right)}
$$

and

$$
u_{0}(x)= \begin{cases}u^{+}\left(x_{0}\right) & \text { if }\left(x, v_{u}\left(x_{0}\right)\right)>0 \\ u^{-}\left(x_{0}\right) & \text { if }\left(x, v_{u}\left(x_{0}\right)\right) \leqslant 0\end{cases}
$$

Since $u_{k} \rightarrow u$ in $L^{1}(\Omega)$, by $(i)$ we get

$$
\lim _{m} \lim _{k} \int_{Q_{v_{u}\left(x_{0}\right)}}\left|u_{m, k}(x)-u_{0}(x)\right| d x=0 .
$$

Moreover, observe that, by (4.30), we get

$$
\lim _{m} \lim _{k} \mu\left(u_{m, k}\right)\left(Q_{v_{u}\left(x_{0}\right)}\right)=z\left(x_{0}\right)
$$

and that inequality (4.31) can be written as

$$
\xi\left(x_{0}\right) \geqslant \lim _{m} \lim _{k} F_{\frac{\varepsilon_{k}}{\rho_{m, k}}}\left(u_{m, k}, Q_{v_{u}\left(x_{0}\right)}\right) .
$$

Let us show now that the mass of $\mu\left(u_{m, k}\right)$ does not concentrate near $\partial Q_{v_{u}\left(x_{0}\right)}$ for $m$ and $k$ large enough.

Given $\delta>0$, by $(i v)$ there exists $\rho(\delta)$ such that $\forall \rho<\rho(\delta)$ and $\forall l \in\{1, \ldots, M\}$ it holds

$$
\rho^{n-1}\left(z_{l}\left(x_{0}\right)-\frac{\delta}{4}\right)<\mu_{l}\left(x_{0}+\rho Q_{v_{u}\left(x_{0}\right)}\right)<\rho^{n-1}\left(z_{l}\left(x_{0}\right)+\frac{\delta}{4}\right) .
$$

Let $m(\delta)$ be such that $\rho_{m}<\rho(\delta) \forall m>m(\delta)$. Then, for every $t \in(0,1]$ there holds

$$
\left(t \rho_{m}\right)^{n-1}\left(z_{l}\left(x_{0}\right)-\frac{\delta}{4}\right)<\mu_{l}\left(x_{0}+t \rho_{m} Q_{v_{u}\left(x_{0}\right)}\right)<\left(t \rho_{m}\right)^{n-1}\left(z_{l}\left(x_{0}\right)+\frac{\delta}{4}\right) .
$$

Thus, for all $t$ such that $|\mu|\left(x_{0}+\partial\left(t \rho_{m} Q_{v_{u}\left(x_{0}\right)}\right)\right)=0$, by (4.35) we get

$$
\begin{aligned}
\mu_{l}\left(x_{0}+\left(\rho_{m} Q_{v_{u}\left(x_{0}\right) \backslash t \rho_{m}} Q_{v_{u}\left(x_{0}\right)}\right)\right) & =\mu_{l}\left(x_{0}+\rho_{m} Q_{v_{u}\left(x_{0}\right)}\right)-\mu_{l}\left(x_{0}+t \rho_{m} Q_{v_{u}\left(x_{0}\right)}\right) \\
& <\rho_{m}^{n-1}\left(z_{l}\left(x_{0}\right)\left(1-t^{n-1}\right)+\frac{\delta}{2}\right) .
\end{aligned}
$$

Let $t(\delta)<1$ be such that for every $l \in\{1, \ldots, M\}$ there holds $z_{l}(0)\left(1-(t(\delta))^{n-1}\right)<\frac{\delta}{2}$. Then, by (4.36), for every $t \in(t(\delta), 1))$ we get

$$
\mu_{l}\left(x_{0}+\left(\rho_{m} Q_{v_{u}\left(x_{0}\right)} \backslash t \rho_{m} Q_{v_{u}\left(x_{0}\right)}\right)\right)<\delta \rho_{m}^{n-1} .
$$

Set $\bar{t}(\delta):=\frac{1+t(\delta)}{2}$ and let $t_{m} \in(t(\delta), \bar{t}(\delta))$ be such that $|\mu|\left(x_{0}+\partial\left(t_{m} \rho_{m} Q_{v_{u}\left(x_{0}\right)}\right)\right)=0$. Thus

$$
\lim _{k} \mu\left(u_{k}\right)\left(x_{0}+\left(\rho_{m} Q_{v_{u}\left(x_{0}\right)} \backslash t_{m} \rho_{m} Q_{v_{u}\left(x_{0}\right)}\right)\right)=\mu\left(x_{0}+\left(\rho_{m} Q_{v_{u}\left(x_{0}\right)} \backslash t_{m} \rho_{m} Q_{v_{u}\left(x_{0}\right)}\right)\right)
$$


and, by (4.37), we may conclude that for any $m>m(\delta)$ there exists $k(m)$ such that for every $l \in\{1, \ldots, M\}$ and $k>k(m)$ there holds

$$
\mu_{l}\left(u_{k}\right)\left(x_{0}+\left(\rho_{m} Q_{v_{u}\left(x_{0}\right)} \backslash \bar{t}(\delta) \rho_{m} Q_{v_{u}\left(x_{0}\right)}\right)\right)<\delta \rho_{m}^{n-1} .
$$

Hence, by (4.38) we infer that for $m$ and $k$ large enough

$$
\mu_{l}\left(u_{m, k}\right)\left(Q_{v_{u}\left(x_{0}\right)} \backslash \bar{t}(\delta) Q_{v_{u}\left(x_{0}\right)}\right)<\delta .
$$

Taking into account (4.32), (4.33), (4.34) and (4.39), by a standard diagonalization procedure we can then find a sequence of positive numbers $s_{j} \rightarrow 0$ and a sequence $w_{j} \in Q_{s_{j}}\left(Q_{v_{u}\left(x_{0}\right)} ; K\right)$ such that $w_{j} \rightarrow u_{0}$ in $L^{1}\left(Q_{v_{u}\left(x_{0}\right)}\right)$ and there holds

$$
\begin{gathered}
\lim _{j} \mu\left(w_{j}\right)\left(Q_{v_{u}\left(x_{0}\right)}\right)=z\left(x_{0}\right), \\
\mu_{l}\left(w_{j}\right)\left(Q_{v_{u}\left(x_{0}\right)} \backslash \bar{t}(\delta) Q_{v_{u}\left(x_{0}\right)}\right)<\delta \forall l \in\{1, \ldots, M\}, \\
\xi\left(x_{0}\right) \geqslant \lim _{j} F_{s_{j}}\left(w_{j}, Q_{v_{u}\left(x_{0}\right)}\right) .
\end{gathered}
$$

Then, by virtue of Lemma 4.9, we can find a sequence $\left(v_{j}\right) \subset \mathbb{B}_{s_{j}}\left(Q_{v_{u}\left(x_{0}\right)} ; K\right)$ such that (4.53) holds and

$$
\xi\left(x_{0}\right) \geqslant \lim _{j} F_{s_{j}}\left(v_{j}, Q_{v_{u}\left(x_{0}\right)}\right) .
$$

Moreover, by (4.40), (4.41) and (4.53) we have that for $j$ large enough

$$
\left|\mu_{l}\left(v_{j}\right)\left(Q_{v_{u}\left(x_{0}\right)}\right)-z_{l}\left(x_{0}\right)\right|<\delta \forall l \in\{1, \ldots, M\} .
$$

Set $T_{j}:=\left[\frac{1}{s_{j}}\right]$ and let $\hat{v}_{j} \in \mathbb{B}_{1}\left(T_{j} Q_{v_{u}\left(x_{0}\right)} ; K\right)$ defined by

$$
\hat{v}_{j}(a):=v\left(s_{j} a\right), \quad a \in \mathbf{Z}^{n} \cap T_{j} Q_{v_{u}\left(x_{0}\right)} .
$$

Then (4.43) implies that for $\mathrm{j}$ large enough

$$
\left|\frac{\# I_{l}\left(\hat{v}_{j}\right)}{T_{j}^{n-1}}-z_{l}\right|<\delta, \quad \forall \in\{1, \ldots, M\}
$$

and (4.42) reads

$$
\xi\left(x_{0}\right) \geqslant \lim _{j} \frac{1}{T_{j}^{n-1}} F_{1}\left(\hat{v}_{j}, T_{j} Q_{v_{u}\left(x_{0}\right)}\right) .
$$

Hence (4.28) immediately follows by the definition of $f_{h o m}$ and by Proposition 4.6.

Step 2. Proof of (4.29).

For $\left|\mu^{s}\right|$-a.e $x_{0} \in \Omega$ we have

(v) $\eta\left(x_{0}\right)=\lim _{\rho \rightarrow 0} \frac{\lambda\left(x_{0}+\rho Q\right)}{\|\mu\|_{1}\left(x_{0}+\rho Q\right)}$,

(vi) $\zeta\left(x_{0}\right)=\lim _{\rho \rightarrow 0} \frac{\mu\left(x_{0}+\rho Q\right)}{\|\mu\|_{1}\left(x_{0}+\rho . Q\right)}$ 
Fix such a $x_{0} \in \Omega$ and let $\left(\rho_{m}\right)$ be a sequence of positive numbers converging to zero such that

$$
\lambda\left(\partial\left\{x_{0}+\rho_{m} Q_{v_{u}\left(x_{0}\right)}\right\}\right)=0, \quad|\mu|\left(\partial\left\{x_{0}+\rho_{m} Q_{v_{u}\left(x_{0}\right)}\right\}\right)=0 .
$$

By (v) and (vi) we get

$$
\begin{aligned}
& \eta\left(x_{0}\right)=\lim _{m} \lim _{k} \frac{\lambda_{k}\left(x_{0}+\rho_{m} Q\right)}{\left\|\mu\left(u_{k}\right)\right\|_{1}\left(x_{0}+\rho_{m} Q\right)}, \\
& \zeta\left(x_{0}\right)=\lim _{m} \lim _{k} \frac{\mu\left(u_{k}\right)\left(x_{0}+\rho_{m} Q\right)}{\left\|\mu\left(u_{k}\right)\right\|_{1}\left(x_{0}+\rho_{m} Q\right)} .
\end{aligned}
$$

We now show that for a suitable sequence $k_{m} \in \mathbf{N}$ the mass of $\lambda_{k_{m}}$ does not concentrate near $\partial\left(x_{0}+\rho_{m} Q\right)$.

By the inner regularity of $\lambda$, given $\delta>0$, for any $\rho>0$ with $\lambda\left(\partial\left(x_{0}+\rho Q\right)\right)=0$ there exists $t(\rho)$ such that for all $t \in[t(\rho), 1]$ there holds

$$
0<\lambda\left(x_{0}+\rho Q\right)-\lambda\left(x_{0}+t \rho Q\right)<\delta\|\mu\|_{1}\left(x_{0}+\rho Q\right) .
$$

Let $t_{m} \in\left[t\left(\rho_{m}\right), 1\right]$ be such that $\lambda\left(\partial\left(x_{0}+t_{m} \rho_{m} Q\right)\right)=0$. Then

$$
\lambda\left(x_{0}+\left(\rho_{m} Q \backslash t_{m} \rho_{m} Q\right)\right)=\lambda\left(x_{0}+\rho_{m} Q\right)-\lambda\left(x_{0}+t_{m} \rho_{m} Q\right)<\delta\|\mu\|_{1}\left(x_{0}+\rho_{m} Q\right) .
$$

In particular, since $\lim _{k} \lambda_{k}\left(x_{0}+\left(\rho_{m} Q \backslash t_{m} \rho_{m} Q\right)\right)=\lambda\left(x_{0}+\left(\rho_{m} Q \backslash t_{m} \rho_{m} Q\right)\right)$ and $\lim _{k}\left\|\mu\left(u_{k}\right)\right\|_{1}\left(x_{0}+\rho_{m} Q\right)=\|\mu\|_{1}\left(x_{0}+\rho_{m} Q\right)$, we have that for $k$ large enough

$$
\lambda_{k}\left(x_{0}+\left(\rho_{m} Q \backslash t_{m} \rho_{m} Q\right)\right)<\delta\left\|\mu\left(u_{k}\right)\right\|_{1}\left(x_{0}+\rho_{m} Q\right) .
$$

Hence, by the previous inequality and by (4.44) and (4.45), we can find a sequence $k_{m}$ such that $\varepsilon_{k_{m}} \ll \rho_{m}$ and

$$
\begin{gathered}
\eta\left(x_{0}\right)=\lim _{m} \frac{\lambda_{k_{m}}\left(x_{0}+\rho_{m} Q\right)}{\left\|\mu\left(u_{k_{m}}\right)\right\|_{1}\left(x_{0}+\rho_{m} Q\right)} \geqslant \lim _{m} \frac{F_{\varepsilon_{k_{m}}}\left(u_{k_{m}}, x_{0}+\rho_{m} Q\right)}{\left\|\mu\left(u_{k_{m}}\right)\right\|_{1}\left(x_{0}+\rho_{m} Q\right)}, \\
\zeta\left(x_{0}\right)=\lim _{m} \frac{\mu\left(u_{k_{m}}\right)\left(x_{0}+\rho_{m} Q\right)}{\left\|\mu\left(u_{k_{m}}\right)\right\|_{1}\left(x_{0}+\rho_{m} Q\right)}, \\
\lambda_{k_{m}}\left(x_{0}+\left(\rho_{m} Q \backslash t_{m} \rho_{m} Q\right)\right)<\delta\left\|\mu\left(u_{k_{m}}\right)\right\|_{1}\left(x_{0}+\rho_{m} Q\right), \\
R \varepsilon_{k_{m}}<\rho_{m}\left(1-t_{m}\right) .
\end{gathered}
$$

Note that the two last inequalities imply that

$$
F_{\varepsilon_{k_{m}}}\left(u_{k_{m}}, x_{0}+\left(\rho_{m} Q \backslash\left(\rho_{m}-R \varepsilon_{k_{m}}\right) Q\right)\right)<\delta\left\|\mu\left(u_{k_{m}}\right)\right\|_{1}\left(x_{0}+\rho_{m} Q\right) .
$$

Observe that, for every $m$ we can find $\tilde{\rho}_{m}$ with $\lim _{m} \frac{\tilde{\rho}_{m}}{\rho_{m}}=1$ and $x_{0}^{m} \in \varepsilon_{k_{m}} \mathbf{Z}^{n}$ with $\lim _{m} x_{0}^{m}=x_{0}$, such that

$$
\varepsilon_{k_{m}} \mathbf{Z}^{n} \cap\left(x_{0}^{m}+\tilde{\rho}_{m} Q\right)=\varepsilon_{k_{m}} \mathbf{Z}^{n} \cap\left(x_{0}+\rho_{m} Q\right)
$$

which in turn implies

$$
\begin{gathered}
F_{\varepsilon_{k_{m}}}\left(u_{k_{m}}, x_{0}+\rho_{m} Q\right)=F_{\varepsilon_{k_{m}}}\left(u_{k_{m}}, x_{0}^{m}+\tilde{\rho}_{m} Q\right), \\
\mu\left(u_{k_{m}}\right)\left(x_{0}+\rho_{m} Q\right)=\mu\left(u_{k_{m}}\right)\left(x_{0}^{m}+\tilde{\rho}_{m} Q\right) .
\end{gathered}
$$


Set $T_{m}:=\frac{\tilde{\rho}_{m}}{\varepsilon_{k_{m}}}$ and let $\hat{u}_{m} \in Q_{1}\left(T_{m} Q ; K\right)$ be defined by

$$
\hat{u}_{m}(a)=u_{k_{m}}\left(x_{0}^{m}+\varepsilon_{k_{m}} a\right) \quad a \in \mathbf{Z}^{n} \cap T_{m} Q .
$$

Note that, by definition, $\left\|\mu\left(\hat{u}_{m}\right)\right\|_{1}(A)=\# I\left(\hat{u}_{m}, A\right)$ for any $A \subset \mathbf{R}^{n}$. Then (4.46) and (4.47) read

$$
\begin{aligned}
& \eta\left(x_{0}\right) \geqslant \lim _{m} \frac{F_{1}\left(\hat{u}_{m}, T_{m} Q\right)}{\# I\left(\hat{u}_{m}, T_{m} Q\right)}, \\
& \zeta\left(x_{0}\right)=\lim _{m} \frac{\mu\left(\tilde{u}_{m}\right)(Q)}{\# I\left(\hat{u}_{m}, T_{m} Q\right)} .
\end{aligned}
$$

Moreover, by (4.48), we get

$$
F_{1}\left(\hat{u}_{m}, T_{m} Q \backslash\left(T_{m}-R\right) Q\right)<\delta \# I\left(\hat{u}_{m}, T_{m} Q\right) .
$$

Hence, (4.29) immediately follows by the definition of $g_{\text {hom }}$, taking into account (4.49), (4.50) and (4.51).

Set, for $v \in S^{n-1}$,

$$
u_{v}= \begin{cases}m_{1} & \text { if }(x, v)>0 \\ m_{2} & \text { if }(x, v) \leqslant 0\end{cases}
$$

Moreover we recall that we have set $Q_{v}=\left(-r_{v}, r_{v}\right)^{n}$.

LEMMA 4.9 Let $s_{j} \rightarrow 0^{+}, v \in S^{n-1}$ and let $w_{j} \in Q_{s_{j}}\left(Q_{\nu} ; K\right)$ be such that $w_{j} \rightarrow u_{v}$ in $L^{1}\left(Q_{\nu}\right)$. Then there exist $v_{j} \in \mathbb{B}_{s_{j}}\left(Q_{\nu} ; K\right)$ such that

$$
v_{j} \equiv u_{v} \text { on } Q_{v} \backslash Q_{j},
$$

where $Q_{j}:=\left(-r_{j}, r_{j}\right)^{n} \subset Q_{\nu}$, for some $r_{j}>0$ such that $\lim _{j} r_{j}=r_{v}$, and

$$
\liminf _{j} F_{S_{j}}\left(w_{j}, Q_{v}\right) \geqslant \liminf _{j} F_{s_{j}}\left(v_{j}, Q_{v}\right) .
$$

Proof. Set

$$
\delta_{j}:=\int_{Q_{v}}\left|w_{j}-u_{v}\right| d x
$$

Let $k_{j} \in \mathbf{N}$ be such that

$$
\frac{\delta_{j}}{s_{j}}<<k_{j}<<\frac{1}{s_{j}}
$$

and set, for $i \in\left\{0, \ldots, k_{j}\right\}$,

$$
\begin{gathered}
r_{j}^{i}:=\left[\frac{r_{\nu}}{s_{j}}\right]+\left(i-k_{j}\right) M, \\
Q_{j}^{i}:=\left(-r_{j}^{i} s_{j}, r_{j}^{i} s_{j}\right) .
\end{gathered}
$$


Then we get

$$
\delta_{j} \geqslant \int_{Q_{v} \backslash Q_{j}^{0}}\left|w_{j}-u_{v}\right| d x \geqslant \sum_{i=0}^{k_{j}-1} \int_{Q_{j}^{i+1} \backslash Q_{j}^{i}}\left|w_{j}-u_{v}\right| d x .
$$

Hence, there exists $i_{j} \in\left\{0, \ldots, k_{j}-1\right\}$ such that, set $S_{j}:=Q_{j}^{i_{j}+1} \backslash Q_{j}^{i_{j}}$, we have

$$
\delta_{j} \geqslant k_{j} \int_{S_{j}}\left|w_{j}-u_{v}\right| d x \geqslant C k_{j} s_{j}^{n} \#\left\{a \in s_{j} \mathbf{Z}^{n} \cap S_{j}: w_{j}(a) \neq u_{v}(a)\right\},
$$

which in turn, by (4.55), implies that

$$
s_{j}^{n-1} \#\left\{a \in \lambda_{j} \mathbf{Z}^{n} \cap S_{j}: w_{j}(a) \neq u_{v}(a)\right\} \rightarrow 0 .
$$

Let, then, $v_{j} \in B_{s_{j}}\left(Q_{\nu} ; K\right)$ defined by

$$
v_{j}(a):= \begin{cases}w_{j}(a) & \text { if } a \in s_{j} \mathbf{Z}^{n} \cap Q_{j}^{i_{j}} \\ u_{v}(a) & \text { otherwise. }\end{cases}
$$

Thus, by (4.56), we get

$$
\begin{aligned}
F_{s_{j}}\left(v_{j}, Q_{v}\right) \leqslant F_{s_{j}}\left(w_{j},\right. & \left.Q_{j}^{i_{j}}\right)+F_{s_{j}}\left(u_{v}, Q_{v} \backslash Q_{j}^{i_{j}}\right) \\
& +C s_{j}^{n-1} \#\left\{a \in s_{j} \mathbf{Z}^{n} \cap S_{j}: w_{j}(a) \neq u_{v}(a)\right\} \leqslant F_{j}\left(w_{j}, Q\right)+o(1),
\end{aligned}
$$

from which we get the conclusion.

Proposition 4.10 ( $\Gamma$-lim sup inequality) We have

$$
\Gamma-\limsup _{\varepsilon \rightarrow 0} F_{\varepsilon}(u, \mu) \leqslant F(u, \mu) .
$$

Proof. We will use the notation $F^{\prime \prime}:=\Gamma$-lim sup $F_{\varepsilon}$. We split the proof in several steps.

Step 1. Claim: (4.57) holds for every $(u, \mu) \in B V\left(\Omega ;\left\{m_{1}, m_{2}\right\}\right) \times\left(m_{+}(\Omega)\right)^{M}$ such that $S(u)$ is a polyhedral set and $\mu$ is of the form $\mu=\varphi \mathcal{H}^{n-1}\left\lfloor S(u)+\sum_{j=1}^{N} w_{j} \delta_{x_{j}}\right.$, where $\varphi: \Omega \rightarrow \mathbf{R}^{M}$ is a piecewise-constant function, $N \in \mathbf{N}$ and, for all $j \in\{1,2, \ldots, N\}, w_{j} \in\left(\mathbf{R}_{+}\right)^{M}$ and $x_{j} \in \Omega$.

Since the construction we provide is local, without loss of generality, we prove the claim in the particular case $u=u_{v}$, and $\mu=z \mathcal{H}_{\lfloor S(u)}^{n-1}+w \delta_{0}$ with $v \in S^{n-1}, z, w \in\left(\mathbf{R}_{+}\right)^{M}$. Here, without loss of generality, we also suppose $0 \in \Omega$. Note that

$$
F(u, \mu)=f_{\text {hom }}(z, v) \mathcal{H}^{n-1}(S(u))+g_{\text {hom }}(\zeta)\|w\|_{1},
$$

where $\zeta=\frac{w}{\|w\|_{1}}$. By the lower semicontinuity of $F^{\prime \prime}$, in order to show (4.57), it suffices to prove that there exists $\left(\mu_{j}\right)_{j} \subset\left(m_{+}(\Omega)\right)^{M}$ weakly converging to $\mu$ as $j \rightarrow+\infty$ such that, for every $j \in \mathbf{N}$, there exists $u_{\varepsilon} \in Q_{\varepsilon}(\Omega ; K)$ such that $\left(u_{\varepsilon}, \mu\left(u_{\varepsilon}\right)\right) \rightarrow\left(u, \mu_{j}\right)$ with respect to the $\tau_{1} \times \tau_{2}$-convergence and

$$
\limsup _{\varepsilon} F_{\varepsilon}\left(u_{\varepsilon}\right) \leqslant F(u, \mu)+\frac{C}{j} .
$$


For simplicity of notation we provide the construction of such $u_{\varepsilon}$ in the case $v=e_{n}$, the same argument applying to the general case. Such a $u_{\varepsilon}$ will be obtained by scaling the periodic extension of an optimal function for the problem defining $f_{h o m}$ in a neighborhood of $S(u)$ and a proper extension of an optimal function for the problem defining $g_{h o m}$ in a suitable neighborhood of 0 .

Let $0<\delta_{j}<\frac{1}{j}, T_{j}>0, u_{j} \in \mathbb{B}_{1}\left(T_{j} Q ; K\right)$ and $v_{j} \in \mathbb{Q}_{1}\left(T_{j} Q ; K\right)$ be such that

$$
\begin{gathered}
\max _{l \in\{1, \ldots, M\}}\left|\frac{\# I_{l}\left(u_{j}, T_{j} Q\right)}{T_{j}^{n-1}}-z_{l}\right| \leqslant \delta_{j}, \max _{l \in\{1, \ldots, M\}}\left|\frac{\# I_{l}\left(v_{j}, T_{j} Q\right)}{\# I\left(v_{j}, T_{j} Q\right)}-\zeta_{l}\right| \leqslant \delta_{j} \\
\frac{1}{T_{j}^{n-1}} F_{1}\left(u_{j}, T_{j} Q\right) \leqslant f_{h o m}\left(z, e_{n}\right)+\frac{1}{j} \\
\frac{F_{1}\left(v_{j}, T_{j} Q\right)}{\# I\left(v_{j}, T_{j} Q\right)} \leqslant g_{h o m}(\zeta)+\frac{1}{j}, \\
\frac{F_{1}\left(v_{j}, T_{j} Q \backslash\left(T_{j}-R\right) Q\right)}{\# I\left(v_{j}, T_{j} Q\right)} \leqslant \delta_{j} .
\end{gathered}
$$

Without loss of generality we choose $T_{j}$ to be an even number. With a little abuse of notation we consider $u_{j}$ to be extended by periodicity to $\mathbf{Z}^{n}$. Moreover we extend $v_{j}$ by reflection with respect to the coordinate axes. More precisely we set $v_{j, 0}=v_{j}$ and, for all $k \in\{1, \ldots, n\}$, we define $v_{j, k}$ recursively as follows: $v_{j, k}$ is the extension of $v_{j, k-1}$ on $\mathbf{R}^{k} \times\left[-\frac{T_{j}}{2}, \frac{T_{j}}{2}\right]^{n-k}$ satisfying the following property

$$
v_{j, k}(a)=v_{j, k}\left(a+\left(T_{j}-2 a_{k}\right) e_{k}\right), \quad \forall a \in \mathbf{R}^{k-1} \times\left[-\frac{T_{j}}{2}, \frac{T_{j}}{2}\right]^{n-(k-1)}+h T_{j} e_{k}, \quad h \in \mathbf{Z} .
$$

We have then obtained that the function $v_{j, n}$ extends $v_{j}$ on all $\mathbf{Z}^{n}$. Let us observe that, by the symmetry hypotheses in (4.2), we have that

$$
F_{1}\left(v_{j, n}, T_{j} Q+h\right)=F_{1}\left(v_{j}, T_{j} Q\right), \quad \forall h \in \mathbf{Z}^{n} .
$$

Let $\tilde{u}_{\varepsilon}: \varepsilon \mathbf{Z}^{n} \rightarrow K$ be defined as

$$
\tilde{u}_{\varepsilon}(a)= \begin{cases}m_{1} & \text { if } a_{n} \geqslant \varepsilon \frac{T}{2} \\ u_{j}\left(\frac{a}{\varepsilon}\right) & \text { if }\left|a_{n}\right| \leqslant \varepsilon \frac{T}{2} \\ m_{2} & \text { if } a_{n} \leqslant-\varepsilon \frac{T}{2}\end{cases}
$$

and set

$$
k_{\varepsilon}=\left[\left(\frac{\|w\|_{1}}{\varepsilon^{n-1} \# I\left(v_{j}, T_{j} Q\right)}\right)^{\frac{1}{n}}\right]
$$

Note that $\tilde{u}_{\varepsilon} \rightarrow u_{v}$ in $L^{1}(\Omega)$ and that $\varepsilon k_{\varepsilon} \rightarrow 0$. We now define $u_{\varepsilon}: \varepsilon \mathbf{Z}^{n} \rightarrow K$ as

$$
u_{\varepsilon}(a)= \begin{cases}v_{j, n}\left(\frac{a}{\varepsilon}\right) & \text { if } a \in \varepsilon k_{\varepsilon} T_{j} Q \\ \tilde{u}_{\varepsilon}(a) & \text { otherwise. }\end{cases}
$$


Then $u_{\varepsilon}$ still converges to $u_{v}$ in $L^{1}(\Omega)$. Moreover, by construction, we have that $\mu\left(u_{\varepsilon}\right) \rightarrow \mu^{j} \in$ $\left(m_{+}(\Omega)\right)^{M}$ where, for $l \in\{1,2, \ldots, M\}$,

$$
\mu_{l}^{j}=\frac{\# I_{l}\left(u_{j}, T_{j} Q\right)}{T_{j}^{n-1}} \mathcal{H}^{n-1}\left\lfloor S(u)+\frac{\# I_{l}\left(v_{j}, T_{j} Q\right)}{\# I\left(v_{j}, T_{j} Q\right)}\|w\|_{1} \delta_{0} .\right.
$$

We can now estimate the energy of $u_{\varepsilon}$. Taking into account the invariance of the energy under integer translations and (4.60), we get

$$
\begin{aligned}
F_{\varepsilon}\left(u_{\varepsilon}\right) \leqslant\left[\frac{\mathcal{H}^{n-1}(S(u))}{\left(T_{j} \varepsilon\right)^{n-1}}\right] \varepsilon^{n-1} F_{1}\left(u_{j}, T_{j} Q\right) & \\
& +k_{\varepsilon}^{n} \varepsilon^{n-1} F_{1}\left(v_{j}, T_{j} Q\right)+C k_{\varepsilon}^{n} \varepsilon^{n-1} F_{1}\left(v_{j}, T_{j} Q \backslash\left(T_{j}-R\right) Q\right)+o(1),
\end{aligned}
$$

where the third term in the right-hand-side is obtained by estimating the energy due to the interactions that cross the boundary of each cube of size $\varepsilon T_{j}$ contained in $\varepsilon K_{\varepsilon} T_{j} Q$. By (4.59) we eventually have

$$
\begin{aligned}
F_{\varepsilon}\left(u_{\varepsilon}\right) \leqslant\left[\frac{\mathcal{H}^{n-1}(S(u))}{\left(T_{j} \varepsilon\right)^{n-1}}\right] \varepsilon^{n-1} T_{j}^{n-1} & \left(f_{\text {hom }}\left(z, e_{n}\right)+\frac{1}{j}\right) \\
& +k_{\varepsilon}^{n} \varepsilon^{n-1} \# I\left(v_{j}, T_{j} Q\right)\left(g_{\text {hom }}(\zeta)+C \delta_{j}+\frac{1}{j}\right)+o(1) .
\end{aligned}
$$

The conclusion follows passing to the limsup as $\varepsilon$ tends to 0 , taking into account (4.61).

Step 2. Claim: (4.57) holds for every $(u, \mu)$ as in Step 1 but with $\varphi \in C\left(\Omega ; \mathbf{R}^{M}\right)$.

Let $\varphi_{k}$ be a sequence of piecewise constant functions such that $\varphi_{k} \rightarrow \varphi$ with respect to the $L^{1}\left(S(u) ; \mathcal{H}^{n-1}\right)$ convergence and let $\mu_{k}=\varphi_{k} \mathcal{H}^{n-1}\left\lfloor S(u)+\sum_{j=1}^{N} w_{j} \delta_{x_{j}}\right.$. Then $\mu_{k} \rightarrow \mu$, and by the convexity and growth properties of $f_{\text {hom }}(\cdot, v)$ stated in Proposition 4.7, $F\left(u, \mu_{k}\right) \rightarrow F(u, \mu)$. Eventually, by the lower semicontinuity of $F^{\prime \prime}(u, \mu)$ and by Step 1, we have

$$
F^{\prime \prime}(u, \mu) \leqslant \liminf _{k} F^{\prime \prime}\left(u, \mu_{k}\right) \leqslant \liminf _{k} F\left(u, \mu_{k}\right)=F(u, \mu) .
$$

Step 3. Claim: (4.57) holds for every $(u, \mu) \in B V\left(\Omega ;\left\{m_{1}, m_{2}\right\}\right) \times\left(m_{+}(\Omega)\right)^{M}$ such that $\mu=$ $\varphi \mathcal{H}^{n-1}\left\lfloor S(u)+\sum_{j=1}^{N} w_{j} \delta_{x_{j}}\right.$ with $\varphi \in C\left(\Omega ; \mathbf{R}^{M}\right)$.

Let $u_{k} \in B V\left(\Omega ;\left\{m_{1}, m_{2}\right\}\right)$ be such that $u_{k} \rightarrow u$ in $L^{1}\left(\Omega ;\left\{m_{1}, m_{2}\right\}\right), S\left(u_{k}\right)$ is a polyhedral set and $\mathcal{H}^{n-1}\left(S\left(u_{k}\right)\right) \rightarrow \mathcal{H}^{n-1}(S(u))$. Let $\mu_{k}=\varphi \mathcal{H}^{n-1}\left\lfloor S\left(u_{k}\right)+\sum_{j=1}^{N} w_{j} \delta_{x_{j}}\right.$. Then we have that $\mu_{k} \rightarrow \mu$ and $|\varphi| \mathcal{H}^{n-1}\left\lfloor S\left(u_{k}\right)(\Omega) \rightarrow|\varphi| \mathcal{H}^{n-1}\left\lfloor S(u)(\Omega)\right.\right.$. Then, by the convexity of $f_{\text {hom }}$ stated in Proposition 4.7 and by Reshetnyak's theorem we have that $F\left(u_{k}, \mu_{k}\right) \rightarrow F(u, \mu)$. Hence we conclude as in Step 2.

Step 4. Claim: (4.57) holds for every $(u, \mu) \in B V\left(\Omega ;\left\{m_{1}, m_{2}\right\}\right) \times\left(m_{+}(\Omega)\right)^{M}$.

Let $\varphi_{k} \in C\left(\Omega ; \mathbf{R}^{M}\right)$ be such that $\varphi_{k} \rightarrow \varphi$ in $\left.L^{1}\left(S(u) ; \mathcal{H}^{n-1}\right)\right)$ and let $\mu_{k}^{s}=\sum_{j=1}^{N} w_{j} \delta_{x_{j}}$ be such that $\mu_{k}^{s} \rightarrow \mu^{s}$ and $\left|\mu_{k}^{s}\right|(\Omega)=\left|\mu^{s}\right|(\Omega)$. Let then $\mu_{k}=\varphi_{k} \mathcal{H}^{n-1}\left\lfloor S(u)+\mu_{k}^{s}\right.$. We have that $\mu_{k} \rightarrow \mu$ and, by the convexity and growth properties of $f_{h o m}(\cdot, v)$ and the convexity of $g_{\text {hom }}$ stated in Proposition 4.7, applying Reshetnyak's theorem we get that $F\left(u_{k}, \mu_{k}\right) \rightarrow F(u, \mu)$. Hence we conclude as in Step 2. 


\subsection{Prescribed volume-fractions}

In this section we study a generalization of the constrained minimum problems introduce at the end of Section 3.2 in the case of the BEG model.

In what follows we set $Q=[0,1)^{n}, \varepsilon_{k}=\frac{1}{k}, k \in \mathbf{N}$ (for simplicity of notation we will drop the $k$ and write $\varepsilon$ instead of $\varepsilon_{k}$ ),

$$
Q_{\varepsilon}^{\#}(Q ; K):=\left\{u: \varepsilon \mathbf{Z}^{n} \rightarrow K: u \text { is } Q \text {-periodic }\right\}
$$

and define $F_{\varepsilon}^{\#}: Q_{\varepsilon}^{\#}(Q ; K) \rightarrow \mathbf{R}$ as

$$
F_{\varepsilon}^{\#}(u)=\sum_{|\xi| \leqslant R} \sum_{a \in Q_{\varepsilon}} \varepsilon^{n-1} f(\xi, u(a), u(a+\varepsilon \xi)) .
$$

Given $\alpha=\left(\alpha_{1}, \alpha_{2}, \ldots, \alpha_{M}\right) \in \mathbf{R}^{M}$ and $\beta \in \mathbf{R}$, let $\alpha_{\varepsilon}=\left(\alpha_{1, \varepsilon}, \alpha_{2, \varepsilon}, \ldots, \alpha_{M, \varepsilon}\right) \rightarrow \alpha$ and $\beta_{\varepsilon} \rightarrow \beta$ as $\varepsilon \rightarrow 0$. We define the set of admissible functions $Q_{\varepsilon}^{\alpha_{\varepsilon}, \beta_{\varepsilon}}(Q ; K)$ as

$$
\begin{array}{r}
Q_{\varepsilon}^{\alpha_{\varepsilon}, \beta_{\varepsilon}}(Q ; K):=\left\{u \in Q_{\varepsilon}^{\#}(Q ; K): \varepsilon^{n-1} \# I_{l}(u, Q)=\alpha_{l, \varepsilon} \forall l \in\{1, \ldots, M\},\right. \\
\left.\varepsilon^{n} \# I_{m_{1}}(u, Q)=\beta_{\varepsilon}\right\},
\end{array}
$$

where we have set $I_{m_{1}}(u, Q):=\left\{a \in Q_{\varepsilon}: u(a)=m_{1}\right\}$, and consider the family of minimum problems

$$
m_{\varepsilon}^{\alpha_{\varepsilon}, \beta_{\varepsilon}}:=\min \left\{F_{\varepsilon}^{\#}(u): u \in Q_{\varepsilon}^{\alpha, \beta}(Q ; K)\right\} .
$$

Note that if $u \in Q_{\varepsilon}^{\alpha_{\varepsilon}, \beta_{\varepsilon}}(Q ; K)$ then $\mu\left(u_{\varepsilon}\right)(Q)=\alpha_{\varepsilon}$. We are interested in studying the limit, as $\varepsilon \rightarrow 0$, of $m_{\varepsilon}^{\alpha, \beta}$. To this end, we introduce the family of functionals $F_{\varepsilon}^{\alpha_{\varepsilon}, \beta_{\varepsilon}}: L_{l o c}^{1}\left(\mathbf{R}^{n} ; K\right) \times$ $\left(m_{+}^{\#}\left(\mathbf{R}^{n}\right)\right)^{M} \rightarrow[0,+\infty]$ defined as

$$
F_{\varepsilon}^{\alpha_{\varepsilon}, \beta_{\varepsilon}}(u, \mu)= \begin{cases}F_{\varepsilon}^{\#}(u) & \text { if } u \in Q_{\varepsilon}^{\alpha_{\varepsilon}, \beta_{\varepsilon}}(Q ; K), \mu=\mu(u), \\ +\infty & \text { otherwise, }\end{cases}
$$

where $u \in Q_{\varepsilon}^{\alpha_{\varepsilon}, \beta_{\varepsilon}}(Q ; K)$ is identified with its piecewise-constant interpolation on the cells of the lattice $\varepsilon \mathbf{Z}^{n}, \mu(u)$ is the surfactant measure defined in (4.6) and $M_{+}^{\#}\left(\mathbf{R}^{n}\right)$ is the space of $Q$ periodic non negative Radon measures. We endow the space $L_{l o c}^{1}\left(\mathbf{R}^{n} ; K\right) \times\left({m_{+}^{\#}}^{*}\left(\mathbf{R}^{n}\right)\right)^{M}$ with the convergence $\tau_{1} \times \tau_{2}$ where $\tau_{1}$ denotes the strong convergence in $L_{l o c}^{1}\left(\mathbf{R}^{n}\right)$ and $\tau_{2}$ denotes the weak*-convergence in $\left(m_{+}\left(\mathbf{R}^{n}\right)\right)^{M}$. Then the following Theorem holds true.

THEOREM 4.11 The family $\left(F_{\varepsilon}^{\alpha_{\varepsilon}, \beta_{\varepsilon}}\right) \Gamma$-converges with respect to the $\tau_{1} \times \tau_{2}$ convergence to the functional $F^{\alpha, \beta}: L_{l o c}^{1}\left(\mathbf{R}^{n}\right) \times\left(m_{+}^{\#}\left(\mathbf{R}^{n}\right)\right)^{M} \rightarrow[0,+\infty]$ defined by

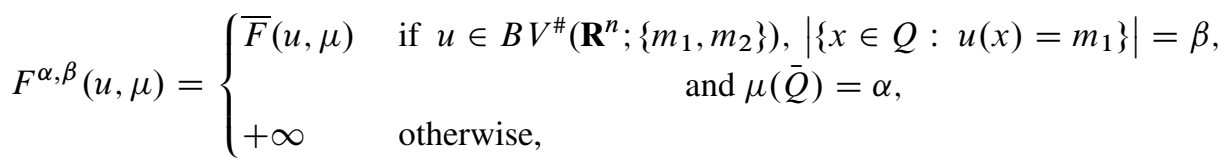

where

$$
\bar{F}(u, \mu)=\int_{S(u) \cap \bar{Q}} f_{\text {hom }}\left(\frac{d \mu}{d \mathcal{H}^{n-1}\lfloor S(u)}, v(u)\right) d \mathcal{H}^{n-1}+\int_{\bar{Q}} g_{\text {hom }}\left(\mu^{s}\right),
$$


$\mu^{s}=\mu-\frac{d \mu}{d \mathfrak{H}^{n-1}\lfloor S(u)} \mathfrak{H}^{n-1}\left\lfloor S(u)\right.$, and the densities $f_{\text {hom }}$ and $g_{\text {hom }}$ are defined in (4.9) and in (4.10).

Proof. It is easy to show that if $u_{\varepsilon} \in Q_{\varepsilon}^{\alpha_{\varepsilon}, \beta_{\varepsilon}}(Q ; K)$ and $\left(u_{\varepsilon}, \mu\left(u_{\varepsilon}\right)\right) \rightarrow(u, \mu)$ with respect to the $\tau_{1} \times \tau_{2}$ convergence, then $(u, \mu) \in B V^{\#}\left(\mathbf{R}^{n} ;\left\{m_{1}, m_{2}\right\}\right) \times\left(m_{+}^{\#}\left(\mathbf{R}^{n}\right)\right)^{M},\left|\left\{x \in Q: u(x)=m_{1}\right\}\right|=$ $\beta$ and $\mu(\bar{Q})=\alpha$. The $\Gamma$-lim inf inequality follows by Theorem 4.4.

The proof of the opposite inequality can be obtained by following the lines of the proof of the $\Gamma$-lim sup inequality of Theorem 4.4, with some extra care to show that the recovery sequence $u_{\varepsilon}$ for $(u, \mu) \in B V^{\#}\left(\mathbf{R}^{n} ;\left\{m_{1}, m_{2}\right\}\right) \times\left(m_{+}^{\#}\left(\mathbf{R}^{n}\right)\right)^{M}$ such that $\mu(\bar{Q})=\alpha$ and $\mid\{x \in Q: u(x)=$ $\left.m_{1}\right\} \mid=\beta$ can be slightly modified so that $u_{\varepsilon} \in \mathbb{Q}_{\varepsilon}^{\alpha_{\varepsilon}, \beta_{\varepsilon}}(Q ; K)$.

As a consequence of the previous Theorem, by the standard properties of $\Gamma$-convergence (we refer the reader to [14] and [23]), we derive the following result about the convergence of the family of minimum problems defined above.

COROLLARY 4.12 There holds

$$
\lim _{\varepsilon} m_{\varepsilon}^{\alpha_{\varepsilon}, \beta_{\varepsilon}}=\min \left\{\bar{F}(u, \mu),\left|\left\{x \in Q: u(x)=m_{1}\right\}\right|=\beta, \mu(\bar{Q})=\alpha\right\} .
$$

Moreover if $\left(u_{\varepsilon}\right) \subset Q_{\varepsilon}^{\alpha_{\varepsilon}, \beta_{\varepsilon}}(Q ; K)$ is such that

$$
\lim _{\varepsilon} F_{\varepsilon}^{\alpha_{\varepsilon}, \beta_{\varepsilon}}\left(u_{\varepsilon}\right)=\lim _{\varepsilon} m_{\varepsilon}^{\alpha_{\varepsilon}, \beta_{\varepsilon}},
$$

then any cluster point $(\bar{u}, \bar{\mu})$ of $\left(u_{\varepsilon}, \mu\left(u_{\varepsilon}\right)\right)$ with respect to the $\tau_{1} \times \tau_{2}$ convergence is a minimizer for $\min \left\{\bar{F}(u, \mu),\left|\left\{x \in Q: u(x)=m_{1}\right\}\right|=\beta, \mu(\bar{Q})=\alpha\right\}$.

REMARK 4.13 The previous Corollary applies to the case of the BEG model, $f(\xi, u, v)$ being defined in (4.5) and the limit energy densities $f_{\text {hom }}$ and $g_{\text {hom }}$ being given by

$$
f_{\text {hom }}(z, v)=\varphi(z, v), \quad g_{\text {hom }}(1)=2(1-k),
$$

with $\varphi$ defined in (3.6).

\section{The Blume-Emery-Griffiths model: Proof of Theorem 3.3}

By Remark 4.2, the functionals $E_{\varepsilon}^{(1)}$ satisfy all the hypotheses of Proposition 4.3 and Theorem 4.4. Hence the compactness result asserted in ( $i$ ) follows by Proposition 4.3. Moreover the integral representation result stated in Theorem 4.4 holds true for the $\Gamma$-limit of $E_{\varepsilon}^{(1)}$. Thus, in order to conclude, it is only left to prove that, for all $(z, v) \in \mathbf{R}^{+} \times S^{1}$,

$$
\begin{aligned}
& f_{\text {hom }}(z, v)=\varphi(z, v), \\
& g_{\text {hom }}(1)=2(1-k) .
\end{aligned}
$$

By a symmetry argument it is sufficient to prove (5.1) for $v_{1}, v_{2}>0$.

Step 1 (lower bounds). In this Step we prove the following two inequalities:

$$
\begin{aligned}
& f_{\text {hom }}(z, v) \geqslant \varphi(z, v), \\
& g_{\text {hom }}(1) \geqslant 2(1-k) .
\end{aligned}
$$


Let us first prove the lower bound for $f_{\text {hom }}$. Without loss of generality we consider $T$ to be an even number. Let $J_{1, T}, J_{2, T}$ be the following sets of integers:

$$
\begin{aligned}
& J_{1, T}=\left\{-\left[\frac{T v_{2}}{2}\right],-\left[\frac{T v_{2}}{2}\right]+1, \ldots,\left[\frac{T v_{2}}{2}\right]-1,\left[\frac{T v_{2}}{2}\right]\right\}, \\
& J_{2, T}=\left\{-\left[\frac{T v_{1}}{2}\right],-\left[\frac{T v_{1}}{2}\right]+1, \ldots,\left[\frac{T v_{1}}{2}\right]-1,\left[\frac{T v_{1}}{2}\right]\right\} .
\end{aligned}
$$

Let $u$ be an admissible test function in the problem defining $f_{\text {hom }}(z, v)$ in (4.9); that is $u \in$ $\mathrm{B}_{\varepsilon}\left(T Q_{\nu} ;\{ \pm 1,0\}\right)$ and

$$
\left|\frac{\# I_{0}\left(u, T Q_{\nu}\right)}{T}-z\right|<\delta
$$

We define

$$
J_{1, T}^{0}(u)=\left\{i \in J_{1, T}: \exists j \in \mathbf{Z} \text { such that }(i, j) \in I_{0}(u)\right\}
$$

and

$$
J_{2, T}^{0}(u)=\left\{j \in J_{2, T}: \exists i \in \mathbf{Z} \text { such that }(i, j) \in I_{0}(u)\right\} .
$$

Note that, by (5.3), for $i \in\{1,2\}$

$$
\# J_{i, T}^{0}(u) \leqslant \# I_{0}\left(u, T Q_{v}\right) \leqslant(z+\delta) T \text {. }
$$

The proof will be the result of the following three estimates.

Estimate (i). By a slicing argument, splitting the energy into the contribution of the horizontal and the vertical interactions, we get

$$
\begin{aligned}
E_{1}^{(1)}(u) \geqslant & 2(1-k) \# J_{1, T}^{0}(u)+2\left(\# J_{1, T}-\# J_{1, T}^{0}(u)\right) \\
& +2(1-k) \# J_{2, T}^{0}(u)+2\left(\# J_{2, T}-\# J_{2, T}^{0}(u)\right) \\
= & -2 k \# J_{1, T}^{0}(u)-2 k \# J_{2, T}^{0}(u)+2\left(\# J_{1, T}+\# J_{2, T}\right) \\
\geqslant & -4 k(z+\delta) T+2\left(2\left[\frac{T v_{2}}{2}\right]+2\left[\frac{T v_{1}}{2}\right]\right) .
\end{aligned}
$$

There follows that

$$
\begin{aligned}
f_{\text {hom }}(z, v) & \geqslant \lim _{\delta \rightarrow 0} \lim _{T \rightarrow+\infty} \frac{1}{T}\left(-4 k(z+\delta) T+2\left(2\left[\frac{T v_{2}}{2}\right]+2\left[\frac{T v_{1}}{2}\right]\right)\right) \\
& =-4 k z+2\left(v_{1}+v_{2}\right) .
\end{aligned}
$$

Estimate (ii). We observe that we may split the energy of the surfactants into two terms. The first term accounts for 2 vertical and 1 horizontal interaction for each column, while the second term accounts for the remaining interaction in each row. By counting the energy due to the non-surfactant 
particles as in estimate (i), we get

$$
\begin{aligned}
E_{1}^{(1)}(u) \geqslant 3(1-k) \# J_{1, T}^{0}(u)+2\left(\# J_{1, T}-\# J_{1, T}^{0}(u)\right) \\
\quad+(1-k) \# J_{2, T}^{0}(u)+2\left(\# J_{2, T}-\# J_{2, T}^{0}(u)\right) \\
\geqslant(1-3 k) \# J_{1, T}^{0}(u)+2 \# J_{1, T} \\
\quad+(1-k) \# J_{2, T}^{0}(u)+(1-k)\left(\# J_{2, T}-\# J_{2, T}^{0}(u)\right) \\
\geqslant(1-3 k)(z+\delta) T+2 \# J_{1, T}+(1-k) \# J_{2, T}(u) \\
\geqslant(1-3 k)(z+\delta) T+4\left[\frac{T v_{2}}{2}\right]+2(1-k)\left[\frac{T v_{1}}{2}\right] .
\end{aligned}
$$

By exchanging the role of $J_{1, T}^{0}(u)$ with that of $J_{2, T}^{0}(u)$ in the previous estimate we have

$$
E_{1}^{(1)}(u) \geqslant(1-3 k)(z+\delta) T+4\left[\frac{T\left(v_{1} \vee v_{2}\right)}{2}\right]+2(1-k)\left[\frac{T\left(\nu_{1} \wedge v_{2}\right)}{2}\right] .
$$

Therefore we get

$$
\begin{aligned}
f_{\text {hom }}(z, v) & \geqslant \lim _{\delta \rightarrow 0} \lim _{T \rightarrow+\infty} \frac{1}{T}(1-3 k)(z+\delta) T+4\left[\frac{T\left(v_{1} \vee v_{2}\right)}{2}\right]+2(1-k)\left[\frac{T\left(v_{1} \wedge v_{2}\right)}{2}\right] \\
& \geqslant(1-3 k) z+2\left(v_{1} \vee v_{2}\right)+(1-k)\left(v_{1} \wedge v_{2}\right) .
\end{aligned}
$$

Estimate (iii). We observe that the we may split the energy of the surfactant into three terms. The first term takes into account two interactions for each surfactant particle. The other two terms take into account in each row and column containing a surfactant at least one interaction between a surfactant and a non-surfactant particle. Counting as in the previous estimates the remaining interactions, we have

$$
\begin{aligned}
E_{1}^{(1)}(u) \geqslant & 2(1-k) \# I_{0}\left(u, T Q_{v}\right)+2\left(\# J_{1, T}-\# J_{1, T}^{0}\right)+(1-k) \# J_{1, T}^{0} \\
& +2\left(\# J_{2, T}-\# J_{2, T}^{0}\right)+(1-k) \# J_{2, T}^{0} \\
\geqslant & 2(1-k)(z-\delta) T+(1-k)\left(\# J_{1, T}+\# J_{2, T}\right) \\
\geqslant & 2(1-k)(z-\delta) T+(1-k)\left(2\left[\frac{T v_{1}}{2}\right]+2\left[\frac{T \nu_{2}}{2}\right]\right) .
\end{aligned}
$$

Hence we have

$$
\begin{aligned}
f_{\text {hom }}(z, v) & \geqslant \lim _{\delta \rightarrow 0} \lim _{T \rightarrow+\infty} \frac{1}{T}\left(2(1-k)(z-\delta) T+(1-k)\left(2\left[\frac{T v_{1}}{2}\right]+2\left[\frac{T v_{2}}{2}\right]\right)\right) \\
& \geqslant 2(1-k) z+(1-k)\left(v_{1}+v_{2}\right) .
\end{aligned}
$$

The lower bound in (5.2) for $g_{\text {hom }}$ is straightforward. In fact, let us observe that, except for a negligible error due to the interactions at the boundary of $T Q$, the energy accounts for at least two interactions of each surfactant particle. Hence, for any test function $u$ in the minimum problem defining $g_{\text {hom }}(1)$ we have

$$
E_{1}^{(1)}(u, T Q) \geqslant 2(1-k) \# I_{0}(u, T Q)+o(1) .
$$


Then

$$
g_{\text {hom }}(1) \geqslant \lim _{\delta} \liminf _{T} \frac{E_{1}^{(1)}(u, T Q)}{\# I_{0}(u, T Q)} \geqslant 2(1-k) .
$$

Step 2 (upper bounds). In this step we conclude the proof by showing that the inequalities

$$
\begin{gathered}
f_{\text {hom }}(z, v) \leqslant \varphi(z, v), \\
g_{\text {hom }}(1) \leqslant 2(1-k) .
\end{gathered}
$$

hold true. With a slight abuse of notation, for $v \in S^{1}$ we denote by $u_{v}: \mathbf{R}^{2} \rightarrow\{ \pm 1\}$ the function defined in (4.52), with -1 and 1 in place of $m_{1}$ and $m_{2}$, that is

$$
u_{v}(x):= \begin{cases}-1 & \text { if }(x, v)>0 \\ +1 & \text { if }(x, v) \leq 0\end{cases}
$$

and, for $z \in \mathbf{R}^{+}$, we set

$$
\mu_{z, v}:=z H^{1}\left\lfloor S\left(u_{v}\right) .\right.
$$

In order to prove the first inequality in (5.4), by Theorem 4.4 it is enough to construct $u_{\varepsilon} \in$ $Q_{\varepsilon}(\Omega ;\{0, \pm 1\})$ such that $\left(u_{\varepsilon}, \mu\left(u_{\varepsilon}\right)\right) \rightarrow\left(u_{v}, \mu_{z, v}\right)$ w.r.t. the $\tau_{1} \times \tau_{2}$-convergence and

$$
\limsup _{\varepsilon} E_{\varepsilon}^{(1)}\left(u_{\varepsilon}\right) \leqslant \varphi(z, v) H^{1}\left(S\left(u_{v}\right) \cap \Omega\right) .
$$

To this end we find it useful to rewrite $\varphi$ as

$$
\varphi(z, v)= \begin{cases}\varphi_{1}(z, v) & \text { if } 0 \leqslant z<\left|v_{1}\right| \wedge\left|v_{2}\right| \\ \varphi_{2}(z, v) & \text { if }\left|v_{1}\right| \wedge\left|v_{2}\right| \leqslant z \leqslant\left|v_{1}\right| \vee\left|v_{2}\right| \\ \varphi_{3}(z, v) & \text { if } z>\left|v_{1}\right| \vee\left|v_{2}\right|\end{cases}
$$

The construction of $u_{\varepsilon}$ differs in the three cases $0<z<\left|v_{1}\right| \wedge\left|v_{2}\right|,\left|v_{1}\right| \wedge\left|v_{2}\right| \leqslant z \leqslant\left|v_{1}\right| \vee\left|v_{2}\right|$ or $z>\left|v_{1}\right| \vee\left|v_{2}\right|$. Without loss of generality, for simplicity of exposition, we may suppose that $-v_{1} \geqslant v_{2}>0$. Moreover, by the continuity of $f_{h o m}(z, \cdot)$ and $\varphi(z, \cdot)$, by a density argument we may assume that $\frac{-v_{1}}{v_{2}} \in \mathbf{Q}$. Let $p, q \in \mathbf{N}$ be such that $\frac{-v_{1}}{v_{2}}=\frac{p}{q}$. By the continuity of $f_{\text {hom }}(\cdot, v)$ and $\varphi(\cdot, v)$ we may further assume that $z^{\prime}:=z \sqrt{p^{2}+q^{2}} \in \mathbf{Q}$. Hence, by possibly replacing $(p, q)$ by $(m p, m q)$ for some $m \in \mathbf{N}$, we may reduce to the case $z^{\prime} \in \mathbf{N}$.

Let us set $\bar{v}=\frac{e_{1}+e_{2}}{\sqrt{2}}$ and let $u^{0}:\{1,2, \ldots, q\} \times \mathbf{Z} \rightarrow\{ \pm 1,0\}$ be defined as

$$
u^{0}(a)= \begin{cases}0 & \text { if } a_{1}=a_{2} \leqslant q \text { or } a_{1}=q, q<a_{2} \leqslant p \\ u_{\bar{v}}(a) & \text { otherwise }\end{cases}
$$

where $a=\left(a_{1}, a_{2}\right)$.

Case 1: $0<z<\left|v_{1}\right| \wedge\left|v_{2}\right|$. By the assumptions on $v_{1}, v_{2}$ this case corresponds to $z^{\prime}<q$. Let $u_{z, v}: \mathbf{Z}^{2} \rightarrow\{ \pm 1,0\}$ be such that

$$
u_{z, v}(a+(p, q))=u_{z, v}(a), \quad \forall a \in \mathbf{Z}^{2}
$$




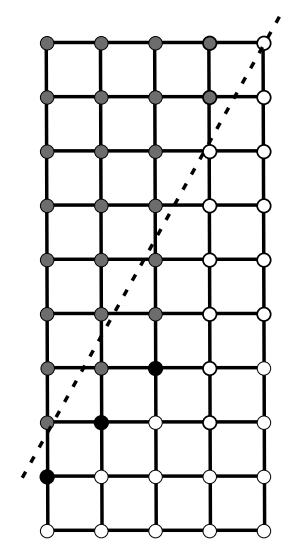

FIG. 6. $u_{z, v}$ in the periodicity cell $\{1,2, \ldots, q\} \times\{1,2, \ldots, p\}$ with $q=5, p=9$ and $z^{\prime}=3$

and, on $\{1,2, \ldots, q\} \times \mathbf{Z} \rightarrow\{ \pm 1,0\}$ is defined as (see Figure 4)

$$
u_{z, v}(a)= \begin{cases}u^{0}(a) & \text { if } 0<a_{1} \leqslant z^{\prime} \\ u_{v} & \text { otherwise }\end{cases}
$$

Let then $u_{\varepsilon}: \varepsilon \mathbf{Z}^{2} \rightarrow\{ \pm 1,0\}$ be such that $u_{\varepsilon}(a)=u_{z, v}\left(\frac{a}{\varepsilon}\right)$. It holds that $\left(u_{\varepsilon}, \mu\left(u_{\varepsilon}\right)\right) \rightarrow$ $\left(u_{v}, \mu_{z, v}\right)$ w.r.t. the $\tau_{1} \times \tau_{2}$-convergence. In order to estimate the energy of $u_{\varepsilon}$ we observe that it concentrates on each rectangle of the type $R_{\varepsilon, j}:=(0, \varepsilon q] \times(0, \varepsilon p]+\varepsilon j(p, q), j \in \mathbf{Z}$ where, by periodicity, it takes the constant value

$$
E_{\varepsilon}^{(1)}\left(u_{\varepsilon}, R_{\varepsilon, j}\right)=\varepsilon\left(4(1-k) z^{\prime}+2\left(p-z^{\prime}\right)+2\left(q-z^{\prime}\right)\right)=\varepsilon\left(-4 k z^{\prime}+2(p+q)\right) .
$$

Then

$$
\begin{aligned}
E_{\varepsilon}^{(1)}\left(u_{\varepsilon}\right) & \leqslant E_{\varepsilon}^{(1)}\left(u_{\varepsilon}, R_{\varepsilon, 0}\right)\left(\#\left\{j \in \mathbf{Z}: R_{\varepsilon, j} \subset \Omega\right\}+2\right) \\
& \leqslant \varepsilon\left(-4 k z^{\prime}+2(p+q)\right)\left(\left[\frac{H^{1}\left(S\left(u_{v}\right) \cap \Omega\right)}{\varepsilon \sqrt{p^{2}+q^{2}}}\right]+2\right) .
\end{aligned}
$$

Eventually, letting $\varepsilon$ tend to 0 we obtain

$$
\limsup _{\varepsilon} E_{\varepsilon}^{(1)}\left(u_{\varepsilon}\right) \leqslant \varphi_{1}(z, v) H^{1}\left(S\left(u_{v}\right) \cap \Omega\right) .
$$

Case 2: $\left|v_{1}\right| \wedge\left|v_{2}\right| \leqslant z \leqslant\left|v_{1}\right| \vee\left|v_{2}\right|$. This case corresponds to $q \leqslant z^{\prime} \leqslant p$. Let $v_{z, v}: \mathbf{Z}^{2} \rightarrow\{ \pm 1,0\}$ be such that

$$
v_{z, v}(a+(p, q))=v_{z, v}(a), \quad \forall a \in \mathbf{Z}^{2}
$$

and, on $\{1,2, \ldots, q\} \times \mathbf{Z} \rightarrow\{ \pm 1,0\}$ is defined as (see Figure 5)

$$
v_{z, v}(a)= \begin{cases}-1 & \text { if } a_{1}=q \text { and } a_{2}>z^{\prime} \\ u^{0} & \text { otherwise }\end{cases}
$$




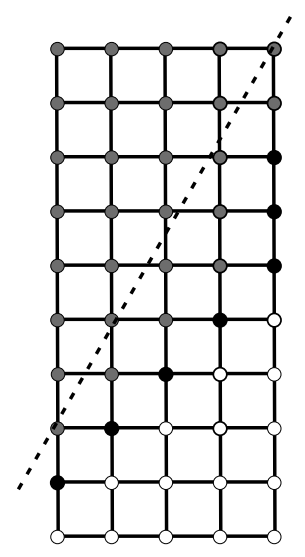

FIG. 7. $u_{z, v}$ in the periodicity cell $\{1,2, \ldots, q\} \times\{1,2, \ldots, p\}$ with $q=5, p=9$ and $z^{\prime}=7$

Let then $v_{\varepsilon}: \varepsilon \mathbf{Z}^{2} \rightarrow\{ \pm 1,0\}$ be such that $v_{\varepsilon}(a)=v_{z, v}\left(\frac{a}{\varepsilon}\right)$. It holds that $\left(v_{\varepsilon}, \mu\left(v_{\varepsilon}\right)\right) \rightarrow$ $\left(u_{v}, \mu_{z, v}\right)$ w.r.t. the $\tau_{1} \times \tau_{2}$-convergence. By arguing as in Case 1, taking into account that

$$
\begin{aligned}
E_{\varepsilon}^{(1)}\left(v_{\varepsilon}, R_{\varepsilon, j}\right) & =\varepsilon\left(4(1-k) q+3(1-k)\left(z^{\prime}-q\right)+2\left(p-z^{\prime}\right)\right) \\
& =\varepsilon\left((1-3 k) z^{\prime}+(1-k) q+2 p\right),
\end{aligned}
$$

we get

$$
E_{\varepsilon}^{(1)}\left(u_{\varepsilon}\right) \leqslant \varepsilon\left((1-3 k) z^{\prime}+(1-k) q+2 p\right)\left(\left[\frac{H^{1}\left(S\left(u_{v}\right) \cap \Omega\right)}{\varepsilon \sqrt{p^{2}+q^{2}}}\right]+2\right) .
$$

Eventually, letting $\varepsilon$ tend to 0 we obtain

$$
\underset{\varepsilon}{\limsup } E_{\varepsilon}^{(1)}\left(v_{\varepsilon}\right) \leqslant \varphi_{2}(z, v) H^{1}\left(S\left(u_{v}\right) \cap \Omega\right) .
$$

Case 3: $z>\left|v_{1}\right| \vee\left|v_{2}\right|$. This case corresponds to $z^{\prime}>p$. Let us extend the function $u^{0}$ to $\mathbf{Z}^{2}$ in such a way that

$$
u^{0}(a+(p, q))=u^{0}(a), \quad \forall a \in \mathbf{Z}^{2} .
$$

We now construct $w_{z, v}: \mathbf{Z}^{2} \rightarrow\{ \pm 1,0\}$ by modifying the function $u^{0}$ suitably increasing the numbers of its zeros in order to match the density constraint on the surfactant phase. More precisely we set $z^{\prime \prime}:=z^{\prime}-\left[\frac{z^{\prime}}{p}\right] p$

$$
\begin{aligned}
& \tilde{I}_{0}:=\bigcup_{m=0}^{\left[z^{\prime} / p\right]}\left(I_{0}\left(u^{0}\right)+m e_{1}\right) \cap \bigcup_{j \in \mathbf{Z}}\left\{a \in \mathbf{Z}^{2}: j p+1 \leqslant a_{2} \leqslant j p+z^{\prime \prime}\right\}, \\
& \hat{I}_{0}:=\bigcup_{m=0}^{\left[z^{\prime} / p\right]-1}\left(I_{0}\left(u^{0}\right)+m e_{1}\right) \cap \bigcup_{j \in \mathbf{Z}}\left\{a \in \mathbf{Z}^{2}: j p+z^{\prime \prime}+1 \leqslant a_{2} \leqslant(j+1) p\right\},
\end{aligned}
$$




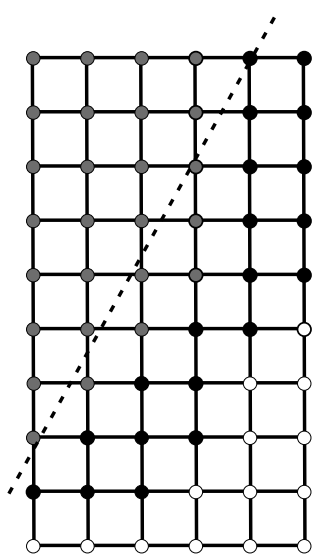

FIG. 8. $u_{z, v}$ in the periodicity cell $\{1,2, \ldots, q\} \times\{1,2, \ldots, p\}$ with $q=5, p=9$ and $z^{\prime}=20$.

and we define (see Figure 6)

$$
w_{z, v}(a)= \begin{cases}0 & \text { if } a \in \tilde{I}_{0} \cup \hat{I}_{0} \\ u^{0} & \text { otherwise }\end{cases}
$$

Let then $w_{\varepsilon}: \varepsilon \mathbf{Z}^{2} \rightarrow\{ \pm 1,0\}$ be such that $w_{\varepsilon}(a)=w_{z, v}\left(\frac{a}{\varepsilon}\right)$. It holds that $\left(w_{\varepsilon}, \mu\left(w_{\varepsilon}\right)\right) \rightarrow$ $\left(u_{v}, \mu_{z, v}\right)$ w.r.t. the $\tau_{1} \times \tau_{2}$-convergence. An easy computation shows that, the energy of each stripe $S_{\varepsilon, j}:=\mathbf{R} \times(j p,(j+1) p], j \in \mathbf{Z}$, is

$$
\begin{aligned}
E_{\varepsilon}^{(1)}\left(w_{\varepsilon}, S_{\varepsilon, j}\right) & =\varepsilon\left((1-3 k) p+(1-k) q+2 p+2(1-k)\left(z^{\prime}-p\right)\right) \\
& =\varepsilon\left((1-k)(p+q)+(1-k) z^{\prime}\right) .
\end{aligned}
$$

Then

$$
\begin{aligned}
E_{\varepsilon}^{(1)}\left(w_{\varepsilon}\right) & \leqslant E_{\varepsilon}^{(1)}\left(w_{\varepsilon}, S_{\varepsilon, 0}\right)\left(\#\left\{j \in \mathbf{Z}: S_{\varepsilon, j} \subset \Omega\right\}+2\right) \\
& \leqslant \varepsilon\left((1-k)(p+q)+(1-k) z^{\prime}\right)\left(\left[\frac{H^{1}\left(S\left(u_{v}\right) \cap \Omega\right)}{\varepsilon \sqrt{p^{2}+q^{2}}}\right]+2\right) .
\end{aligned}
$$

Eventually, letting $\varepsilon$ tend to 0 we obtain

$$
\underset{\varepsilon}{\lim \sup } E_{\varepsilon}^{(1)}\left(w_{\varepsilon}\right) \leqslant \varphi_{3}(z, v) H^{1}\left(S\left(u_{v}\right) \cap \Omega\right) .
$$

We now prove the second inequality in (5.4). To this end, by Theorem 4.4, given $x_{0} \in \Omega$, it is enough to construct a sequence of functions $u_{\varepsilon} \in Q_{\varepsilon}(\Omega ;\{0, \pm 1\})$ such that $\left(u_{\varepsilon}, \mu\left(u_{\varepsilon}\right)\right) \rightarrow\left(1, \delta_{x_{0}}\right)$ w.r.t. the $\tau_{1} \times \tau_{2}$-convergence and that

$$
\limsup _{\varepsilon} E_{\varepsilon}^{(1)}\left(u_{\varepsilon}\right) \leqslant 2(1-k)
$$


We set

$$
u_{\varepsilon}(a)= \begin{cases}0 & \text { if } a \in\left(x_{0}+\left(-\frac{\sqrt{\varepsilon}}{2}, \frac{\sqrt{\varepsilon}}{2}\right)^{2}\right) \cap \varepsilon \mathbf{Z}^{2}, \\ 1 & \text { otherwise. }\end{cases}
$$

Let us observe that $\varepsilon \# I_{0}\left(u_{\varepsilon}\right)=1+o(1)$ and that, each surfactant particle whose interactions do not cross the boundary of $x_{0}+\left(-\frac{\sqrt{\varepsilon}}{2}, \frac{\sqrt{\varepsilon}}{2}\right)^{2}$ gives a contribution to the energy which is equal to $2(1-k)$. Moreover, since the number of the remaining surfactants scales as $\frac{1}{\sqrt{\varepsilon}}$, we have

$$
E_{\varepsilon}^{(1)}\left(u_{\varepsilon}\right)=\varepsilon 2(1-k) \# I_{0}\left(u_{\varepsilon}\right)+o(1)=2(1-k)+o(1)
$$

Letting $\varepsilon$ tend to 0 we get the conclusion.

\section{REFERENCES}

1. ACERbi, E. \& Bouchitté, G. A general class of phase transition models with weighted interface energy. Ann. I H. Poincaré-AN 25 (2008), 1111-1143. Zbl1169. 35367 MR2466324

2. Alicandro, R., Braides, A. \& Cicalese, M. Phase and anti-phases boundaries in binary discrete systems: a variational viewpoint. Netw. Heterog. Media 1 (2006), 85-107. Zbl1131. 49029 MR2219278

3. Alicandro, Braides R. A. \& Cicalese, M. Continuum limits of discrete thin films with superlinear growth densities. Calc. Var. Partial Differential Equations 33 (2008) 267-297. Zbl1148. 49010 MR2429532

4. AlicAndro, R. \& CiCAlese, M. Representation result for continuum limits of discrete energies with superlinear growth. SIAM J. Math Anal. 36 (2004), 1-37. Zbl1070. 49009 MR2083851

5. Alicandro, R. \& Cicalese, M. Variational analysis of the asymptotics of the $X Y$ model. Arch. Rat. Mech. Anal. 192 (2009), 501-536. Zbl1171.82004 MR2505362

6. Alicandro, R., CiCAlese, M. \& Gloria, A. Integral representation of the bulk limit of a general class of energies for bounded and unbounded spin systems. Nonlinearity 21 (2008), 1881-1910.

7. Alicandro, R., Cicalese, M. \& Gloria, A. Integral representation results for energies defined on stochastic lattices and application to nonlinear elasticity. Arch. Ration. Mech. Anal. 200 (2011), 881-943. Zbl pre05952990 MR2796134

8. Alicandro, R. , Cicalese, M. \& Ponsiglione, M. Variational equivalence between GinzburgLandau, XY spin systems and screw dislocations energies. Indiana Univ. Math. J. (to appear).

9. Ambrosio, L. \& Braides, A. Functionals defined on partitions of sets of finite perimeter, I and II. $J$. Math. Pures. Appl., 69 (1990), 285-305 and 307-333. Zb10676 . 49029 MR1070482

10. Ambrosio, L. , Fusco, N. \& Pallara, D. Functions of Bounded Variation and Free Discontinuity Problems. Oxford University Press, Oxford (2000). Zb10957 . 49001 MR1857292

11. X. Blanc, C. Le Bris, P.L. Lions, From molecular models to continuum mechanics. Arch. Rational Mech. Anal. 164 (2002), 341-381. Zbl1028.74005 MR1933632

12. Blanc, X., Le Bris, C. \& Lions, P.L. Atomistic to continuum limits for computational materials science. M2AN Math. Model. Numer. Anal. 41 (2007), 391-426. Zbl1144. 82018 MR2339634

13. Blume, M., Emery V.J. \& GRIfFiths, R.B. Ising Model for the $\lambda$ Transition and Phase Separation in $\mathrm{He}^{3}-\mathrm{He}^{4}$ Mixtures. Physical Review A 4 (1971), 1071-1077.

14. Braides, A. Г-Convergence for Beginners, Oxford University Press, Oxford, 2002. Zb11198.49001 MR1968440

15. BRAIDES, A. A handbook of $\Gamma$-convergence. In: Handbook of Differential Equations. Stationary Partial Differential Equations, Volume 3 (M. Chipot and P. Quittner, eds.) Elsevier 2006. Zb11195. 35002 
16. Braides, A. \& CiCAlese, M. Surface energies in nonconvex discrete systems. Math. Models Methods Appl. Sci. 17, (2007), 985-1037. Zbl1205.82036 MR2337429

17. Braides, A., LEw, A.J. \& ORTIZ, M. Effective cohesive behavior of layers of interatomic planes. Arch. Rational Mech. Anal. 180 (2006), 151-182. Zbl1093.74013 MR2210908

18. Braides, A. \& Piatnitski, A. Overall properties of a discrete membrane with randomly distributed defects. Arch. Ration. Mech. Anal. 189 (2008), 301-323. Zbl1147.74039 MR2413098

19. Braides, A. \& Piatnitski, A. Variational problems with percolation: Dilute spin systems at zero temperature, Preprint 2011.

20. Braides, A. \& Piatnitski, A. Homogenization of surface and length energies for spin systems, Preprint 2010.

21. Braides, A. \& Truskinowsky, L. Asymptotic expansions by $\Gamma$-convergence. Cont. Mech. Therm. 20 (2008), 21-62. Zbl1160.74363 MR2398821

22. CiACh, A., Hoye, J.S. \& Stell, G. Microscopic model for microemulsions. I. Ground states properties. J. Chem. Phys. 90 (1989), 1214-1221.

23. Dal Maso, G. An Introduction to $\Gamma$-Convergence, Progress in Nonlinear Differential Equations and their Applications, 8. Birkhäuser Boston, Inc., Boston, MA (1993). Zbl0816. 49001 MR1201152

24. Ellis, R. S., Otto, P. T. \& Touchette, H. Analysis of phase transitions in the mean-field BlumeEmery-Griffiths model. Ann. Appl. Probab. 15 (2005) 2203-2254. Zbl1113. 82017 MR2152658

25. Fonseca, I., Morini, Y. \& Slastikov, V. Surfactants in foam stability: A phase field model. Arch. Rat. Mech. Anal. 183 (2007), 411-456. Zbl1107.76076 MR2278411

26. Gomprer, G. \& Schick, M. Self-Assembling Amphiphilic Systems, Academic Press, London (1994).

27. Laradji, M., Guo, H., Grant, M. \& Zuckermann, M.J. Phase diagram of a lattice model for ternary mixtures of water, oil, and surfactants. J. Phys. A 24 L629 (1991).

28. Laradji, M., Guo, H., Grant, M. \& Zuckermann, M.J. The effect of surfactants on the dynamics of phase separation. J. Phys. Condens. Matter 46715 (1992).

29. Rosen, M. J. Surfactants and Interfacial Phenomena, Wiley, New York (1989).

30. Ponsiglione, M. Elastic energy stored in a crystal induced by screw dislocations: From discrete to continuous, SIAM J. Math. Anal. 39 (2007), 449-469. Zbl1135.74037 MR2338415

31. SCHICK, M. \& SHIH, W. H. Simple microscopic model of a microemulsion. Phys. Rev. Letters 5911 (1987), 1205-1208.

32. Schmidt, B. On the passage from atomic to continuum theory for thin films. Arch. Ration. Mech. Anal. 190 (2008) 1-55. Zbl1156.74028 MR2434899 